\title{
BotAF, a new Buthus occitanus tunetanus scorpion toxin, produces potent analgesia in rodents
}

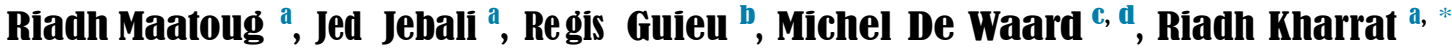 \\ ${ }^{a}$ Universite de Tunis El Manar, Institut Pasteur de Tunis, Laboratoire des Venins et Biomolecules Th erapeutiques, 13, Place Pasteur BP-74, Tunis, 1002 \\ Tunisia \\ b Biochimie, Heital de la Timone, 13005, Marseille, France \\ Inserm U1087, Institut du Thorax, groupe Ilh, Universite de Nantes, 8 quai moncousu, 44000, Nantes, France \\ d Smartox Biotechnology, 570 rue de la chimie, bàiment Nanobio, 38700, Saint Martin d'Hères, France
}

\section{Article history:}

Received 31 August 2017

Received in revised form

8 January 2018

Accepted 9 January 2018

Available online $\mathbf{x x}$

Keywords:

Bio-guided screening

Long chain scorpion toxins

Antinociception

Analgesia

Venom

Buthus occitanus tunetanus

\begin{abstract}
a b s t r a c t
This work reports the purification of new potent scorpion neuropeptide, named Botaf, by an activityguided screening approach. BotaF is a 64-residue long-chain peptide that shares very high similarity with the original b-like scorpion toxin group, in which several peptides have been characterized to be anti-nociceptive in rodents. BotaF administration to rodents does not produce any toxicity or motor impairment, including at high doses. In all models investigated, Botaf turned out to be an efficient peptide in abolishing acute and inflammatory (both somatic and visceral) pain in rodents. It periorms with high potency compared to standard analgesics tested in the same conditions. The anti-nociceptive activity of Botaf depends on the route of injection: it is inactive when tested by i.c.v. or i.v. routes but gains in potency when pre-injected locally (in the same compartment than the irritant itseli) or by i.t. root 40 to 60 min before pain induction, respectively. BotAF is not an AINS-like compound as it fails to reduce inflammatory edema. Also, it does not activate the opioidergic system as its activity is not afiected by naloxone. Botaf does also not bind onto RyR and has low activity towards DRG ion channels (particularly TTX sensitive $\mathrm{Na}^{\mathrm{b}}$ channels) and does not bind onto rat brain synaptosome receptors. In somatic and visceral pain models, Botaf dose-dependently inhibited lumbar spinal cord $\mathrm{c}$-ios/c-jun mRNA up regulation. Altogether, our data favor a spinal or peripheral anti-nociceptive mode of action of BotAF.
\end{abstract}

\section{Introduction}

Animal venoms are reliable sources for drug discovery and implementation of new pharmaceutical tools (Attarde et al., 2016). The richness and complexity in venom composition is the result of Darwinian selection forces onto several genes constituting families of coding toxins. As a result of this evolutionary pressure, the selected natural compounds act on an as diverse number of biological targets that include ion channels, G-protein coupled receptors, transporters and enzymes. Most often, these venom components bind with exquisite selectivity and potency on their receptors making venom-derived components highly attractive pharmacological tools and possible drug leads. The usefulness of

\footnotetext{
corresponding author.

E-mail address: riadh.kharrat@pasteur.tn (R. Kharrat).
}

venomous compounds as analgesics has been validated in several preclinical studies. This is the case of the following compounds: uconotoxin MVIIA (ziconotide) (Essack et al., 2012), p-Theraphotoxin-Pc1a (PcTx1) (Escoubas et al., 2003), APETx2 from a sea anemone species (Jensen et al., 2014), and long neurotoxin 0H-55 (hannalgesin) (Pu et al., 1995). These analgesic compounds arise from difierent venom sources and species (conus, snake, sea anemone, spiders) and were discovered by using diverse screening strategies as well (target-based, toxin-based and activity-based). While many anti-pain compounds have been identífied so far, ziconotide remains the only toxin-derived therapeutic for pain treatment that reached the clinics. This difficulty in converting preclinical leads into therapeutics may be the refiection of two major challenges: i) reaching proper potency and selectivity and ii) providing proper target accessibility and coverage. Part of these challenges may be met by further discovering novel compounds or simply by improving the potency, selectivity and delivery of known 
anti-pain toxins. As such, expanding the screening efiorts to scorpion venom toxins may represent a promising approach. To date, most of the investigators interest has focused on long-chain insectotoxins based on the assumption that they should lack toxicity in mammals and on the well-documented analgesic potential of their targeted sodium channels in pain physiology. Indeed, this is the case for BmKIT2 (Guan et al., 2001), BmkAs (Cui et al., 2010), LqqIT2 (Martin-Eauclaire et al., 2010) and BmKAEP (Wang et al., 2011). These compounds are purified by an in vivo screening approach of abundant venom compounds (or at least highly active compounds) checking for phenotypic or behavioral changes. Relevant compounds isolated this way are further investigated for identifying their molecular targets and for assessing their pharmacodynamics by in vitro approaches. While targetbased drug discovery allows for the identification of new molecular tools of interest in pharmacology, it may end up providing frustrating results in vivo because of the complexity of pain etiology, the challenges faced with the pharmacokinetics and the importance of the mode of administration, with or without adequate formulation. In contrast, compounds isolated against a rigorously defined in vivo pain model should deliver compounds that meet the criteria of preclinical evaluation, with yet uncertainties regarding the mode of action and the molecular target. The main advantage nevertheless remains that the compounds isolated through phenotypic assays will be more easily characterized with regard to the pharmacodynamics parameters (efficacy, potency, delivery...).

Herein, we used an activity-guided screening strategy to identify a venom compound from a Tunisian scorpion using an in vivo rodent pain model. We periormed a double in vivo screening by assessing both toxicity and analgesia of the fractionated venom compounds. Next, we investigated some of its basic pharmacological properties in vivo by using standardized pain models and ruled out some possible anti-nociceptive molecular targets.

\section{Materials and methods}

\subsection{Animals}

All anti-nociceptive experiments were conducted on healthy adult male c57/BL6 mice (25e30 g) and adult female Whistar rats (180e220 g). For toxicity tests, male c57/BL6 mice (18e22 g) were used. Animals were housed at a density of 6 per cage and had free access to food and water in a quiet environment. The room was maintained at $21 \mathrm{e23}{ }^{\circ} \mathrm{C}$ with a $12 \mathrm{~h}$ light/dark cycle. The experimenter was blind to the pharmacological treatment. All animal procedures were reviewed and approved by the Ethics committee of the Pasteur Institute of Tunis according to the guidelines on the use of living animals in scientific investigations. All efiorts were made to minimize both the suffering and number of animal used.

\subsection{Purífication procedure}

$120 \mathrm{ml}$ of crude venom of the scorpion Buthus occitanus tunetanus (Bot) was obtained by electrical milking of field-collected scorpions at the Veterinarian Laboratory of the Institute Pasteur of Tunis. This crude venom of Bot was water extracted, then submitted to gel filtration chromatography on sephadex 650 columns (2 $x$ K26/50, GE Healthcare, France) equilibrated with $0.1 \mathrm{M}$ acetic acid. Five fractions (MI, MII, BotG50, III and VI) were resolved. Fractions MII and BotG50 were lyophilized, resuspended in water and further fractionated by a reversed-phase HPIC (HewlettePackard Series II 1100 liquid chromatography with diode array UV detection) equipped with a semi-preparative c8 column (10 mm x $250 \mathrm{~mm}, 5 \mathrm{~mm}$, Beckman Fullerton, USA). For this purpose, buffer A was $0.1 \%$ trífluoroacetic acid in water and buffer B was $0.1 \%$ triffuoroacetic acid (TFA) in acetonitrile. The column effluents were monitored at 280 and $214 \mathrm{~nm}$. Peptide fractions were eluted at $1 \mathrm{~mL} / \mathrm{min}$ in a linear gradient of bufier $B$ in buffer $A$ and at fixed $25{ }^{\circ} \mathrm{C}$ temperature. Fraction F28, was loaded onto an analytical C18 reversed-phase HPIC column $(10 \mathrm{~mm} \times 250 \mathrm{~mm}, 5 \mathrm{~mm}$, Beckman Fullerton, USA) using a linear gradient of bufier B in buffer A allowing the isolation of Botaf. Protein concentration was measured by amino-acid analysis.

\subsection{Mass spectrometry}

Samples were analyzed on a Voyager-DETM PRO MALDIeTOF (matrix-assisted laser-desorption ionizationetime-of-flight) Workstation mass spectrometer (Perceptive Biosystems, USA). The peptide was dissolved in $30 \%$ acetonitrile with $0.3 \%$ TFA to obtain a concentration of 1 e10 $\mathrm{pmol} / \mathrm{mL}$. The matrix was prepared as iollows: a-cyanohydroxycinnamic acid was dissolved in $50 \%$ acetonitrile in $0.3 \% \mathrm{TFA} / \mathrm{H}_{2} \mathrm{O}$ to obtain a saturated solution at $10 \mathrm{mg} / \mathrm{mL}$. $\mathrm{A}$ $0.5 \mathrm{~mL}$ of peptide solution was then mixed with $0.5 \mathrm{~mL}$ of matrix solution and placed on the sample plate, and the mixture was allowed to dry. Mass spectra were recorded in reffectron mode aiter external calibration with suitable standards and were analyzed using the GRAMS/386 software (Galactic Industries Corporation).

\subsection{Reduction and alkylation of peptide}

Five nanomoles of Botaf was dissolved in $40 \mathrm{~mL}$ of alkylation bufier (6 M guanidine HCl, $0.5 \mathrm{M}$ Tris/HCl, 2 mM EDTA, pH 7.5) containing $1.4 \mathrm{mmol}$ dithiothreitol (DTT). After $1 \mathrm{~h}$ of reduction at $37^{\circ} \mathrm{C}, 9 \mathrm{mmol}$ of 4 -vinylpyridine (4-VP) was added for alkylation. The S-alkylated BotAF (SA-BotAF) was then desalted by dialyze against $10 \%$ acetic acid at $4{ }^{\circ} \mathrm{C}$ overnight using spectra/Por 6 membrane tubing with a 4000 MW cut-ofi (Spectrum Laboratories Inc., USA). The dialyzed product was then subjected to SDS-PAGE on a $15 \%$ gel and then revealed by Coomassie Blue staining.

\subsection{In-gel digestion of alkylated BotAF by trypsin and S. aureus V-8 protease}

The in-gel digestion of SA-BotAF was periormed as previously described (Jeno Paul et al., 1995). Briefly, SDS-PAGE gel protein bands were excised after coomassie Blue distaining, then washed with 40\% n-propanol followed by $0.2 \mathrm{M} \mathrm{NH}_{4} \mathrm{HCO}_{3} / 50 \%$ acetonitrile, and finally dried in Speed Vac. Gel pieces were rehydrated in $100 \mathrm{~mL}$ of $100 \mathrm{mM} \mathrm{NH}_{4} \mathrm{CO}_{3}$ pH 7.8 containing $1 \mathrm{mg}$ trypsin or S. aureus V8 protease. Aiter $24 \mathrm{~h}$ of digestion at $37^{\circ} \mathrm{C}$, peptide fragments were extracted three times from the gel slices with three consecutive treatments: first, $200 \mathrm{~mL}$ of $0.1 \%$ TFA, 0.01\% Tween 20; second, $100 \mathrm{~mL}$ of $1 \%$ TFA, $0.01 \%$ Tween 20; and third, $100 \mathrm{~mL}$ of $0.1 \%$ TFA, 50\% acetonitrile, 0.01\% Tween20 (Eckerskorn and Lottspeich, 1990). Enzymatic digests were separated by high-periormance liquid chromatography on an analytical C18 reverse phase HPIC column.

\subsection{Amino acid sequencing and sequence analyses}

The amino acid sequences of the $\mathrm{N}$-terminal fragment and of the peptides derived from proteolytic digests of SA-BotAF were determined by an automatic liquid-phase protein sequencer (model 476A, Applied Biosystems, USA) using a standard Edman protein degradation procedure (Edman and Begg, 1967). Search for proteins homologous to BotAF was periormed using the NCBI BLAST database search program implemented at the web site (htip://www. ncbi.nlm.nih.gov/). The amino acid sequence of BotaF was aligned with other toxins using Clustal W (Thompson et al., 1994) running 
in BíoEdit version 7.2.6 (Hall, 1999).

\subsection{Cloning of BotaF isoforms}

Total RNA were extracted from homogenized Bot scorpion telsons, containing venomous glands, using the RNAgents kit from Promega (Madison, WI, USA). 5 Bot scorpions were used for this purpose. Single stranded cDNA was obtained by reversedtranscription using an universal oligo(dT)-containing adapter primer $\quad 5^{1}$-GGCCACGCGTCGaCtagtaC(dT) $)_{17}-3^{1}$, Proligo). The following reaction mixture: $12 \mathrm{~mL}$ of DEPC (diethylpyrocarbonate)treated water containing $0.1 \mathrm{mg}$ of primer and $1 \mathrm{mg}$ of total venom gland RNA, 2 mL of 0.1 M DTT, 1 mL of RNAsin (ribonuclease inhibitor at 40 units/mL from Promega), $1 \mathrm{~mL}$ of dNTP (10 mM each, Promega) and 200 units of M-MIV RT (Moloney murine leukaemia virus reverse transcriptase). The final volume was adjusted to $20 \mathrm{~mL}$, and the reaction mixture was incubated at $37^{\circ} \mathrm{C}$ for $1 \mathrm{~h}$, followed by 10 min at $95{ }^{\circ} \mathrm{C}$ to inactivate the enzyme. At the end of the reaction, RNAse H was added to degrade mRNA.

Nexi, cDNA sequence encoding BotaF analogues were amplífied by PCR using venom gland CDNA as template and the following primers: a forward primer corresponding to a degenerated sequence $\quad\left(5^{0}\right.$-GAT-AA(T/C)-GGT-TA(T/C)-GT(N)-CTT-GAC-3) derived from the $\mathrm{N}$-terminal peptide sequence of BotaF and also based on the CDNA sequence homology of three homologous peptides of BotaF (BmKAS, BmKAS1 and BotIT2), and a reverse primer (5 $^{0}$ - GGC-CAC-GCG-TCG-ACT-AGT-AC-3 $\left.{ }^{\prime}\right)$, corresponding to the universal amplification primer used for cDNA synthesis. The PCR protocol included an initial denaturizing step at $95{ }^{\circ} \mathrm{C}$ ior 2 min followed by 35 cycles of denaturation (30 s at $94^{\circ} \mathrm{C}$ ), annealing (30 s at $52{ }^{\circ} \mathrm{C}$ ) and extension (30 s at $72{ }^{\circ} \mathrm{C}$ ), and a final extension for 7 min at $72{ }^{\circ} \mathrm{C}$.

The amplified fragments were separated on $1 \%$ agarose gel electrophoresis. Desired bands were excised under UV-light and purified using the Gel Extraction Kit (QIAGEN) and were cloned into DGEM-T vector (Promeda). Positive recombinant clones were confirmed by PCR-amplífication and sequencing.

\subsection{Toxicity test and rotarod test}

Botaf was tested for in vivo toxicity on $20 \pm 2 \mathrm{~g}$ male mice c57/ BL6 by intracerebroventricular (i.c.v.) injection of $7 \mathrm{~mL}$ of saline solution containing increasing amounts of the peptides. Six mice were used for each concentration. Behavioral parameters considered as signs of toxicity were: tremors, convulsions, paralysis, upward tail and fur, abnormal gait and posture (Clot-Faybesse et al., 2001; Galeotti et al., 2003). Mortalíty was assessed $48 \mathrm{~h}$ after î.c.v. administration.

Accelerating rotarod test (Jones and Roberts, 1968) was assessed prior to pain behaviors study to further check if there is any motor impairment due to BotAF injection. The rotarod apparatus (Ugo Basile Biological Research Apparatus, Varese, Italy) is used as described (Plummer et al., 1991). Briefly, male C57/BL6 mice (18e22 g) were placed on the rod when it was rotating at a speed of $2.5 \mathrm{rpm}$. Aiter 10 set, the timer was started and the speed of the rod was increased (2.5e25 rpm over 5 min). Four Botaf-treated groups (6 mice per group) were tested: (1, $2 \mathrm{mg} / \mathrm{kg})$ i.p. and $(0.2,0.5 \mathrm{mg} / \mathrm{kg})$ i.t.. The drop latency for each tested animal groups was recorded before, $30 \mathrm{~min}$, and $60 \mathrm{~min}$ aiter treatments. Evaluation of BotAF action on motor impairment was assessed by comparing, for each group, the drop latency recorded beiore and 30 min or 60 min aiter treatment.

\subsection{Intrathecal injection}

Intrathecal administration was done by a direct injection on conscious rat and mice as described previously (Mestre et al., 1994). Briefly, the injections were periormed by a 25-Ga $x 1^{11}$ needle, connected to a 25-1xL Hamilton syringe, into the tissues between the dorsal aspects of $\mathrm{L} 5$ and $\mathrm{L6}$, perpendicular to the vertebral column. This site was selected so that the injection was restricted to the region where the spinal cord ends and the cauda equina begins in order to reduce the possibility of spinal damage and increase the intervertebral accessibility. The syringe was held in position for a few seconds and progressively removed to avoid any outflow of the drug.

\subsection{Pain models}

All injected drugs were dissolved in saline. Volumes for mice injection were: $7 \mathrm{~mL}$ by i.c.v., $150 \mathrm{~mL}$ intraperitoneally (i.p.), $15 \mathrm{~mL}$ by intrathecal route (i.t.) and $50 \mathrm{~mL}$ by intraplantar (i.pl.). Each animal was tested only once and sacrificed thereaiter. Several pain models were used: the hot-plate test, the writhing test, the tail flick test and the formalin test, as briefiy described hereunder.

The writhing test consists of an i.p. injection of acetic acid $\mathbf{( 0 . 6 \%}$, $7.5 \mathrm{mg} \mathrm{kg}^{-1}$ ) 10 mice pretreated at different time before with the compounds of interest. Results are presented as inhibition percent of the contortion numbers that occur during 30 min following the injection of acetic acid (Collier et al., 1968).

For the hot-plate test, mice are placed into a glass cylinder $\left(14 \mathrm{~cm}\right.$ diameter and $20 \mathrm{~cm}$ height) on a hot-plate adjusted to $55^{\circ} \mathrm{C}$ (hot-plate analgesia meter, Harvard Apparatus, USA). The latency of the hind paw licking was used as an index of pain reaction. A $60 \mathrm{~s}$ cut-off was used to preserve the physical integrity of the animal.

Concerning the tail filck test, the apparatus consists of a shuttercontrolled lamp as a heat source (tail fick analgesia meter, Harvard Apparatus, USA). The test was periormed as described previously (D'Amour and Smith, 1941). Briefly, the tail of the mice was marked at a distance of $2 \mathrm{~cm}$ from the apex and was placed under the heat source. The time taken by the treated animal to fick its tail was recorded. A cut-off of $60 \mathrm{~s}$ was used to respect animal integrity.

For the formalin test, $50 \mathrm{~mL}$ of $\mathbf{5 \%}$ formalin was administered i.pl. into the leit hind paw. Each rat was placed individually in a plexiglass test box for at least 30 min to adapt to the new environment before administration of the chemical agents. Nociceptive behaviors were determined by counting the time spent in liking and finching the injected hind-paw over consecutive periods of 5 min during $1 \mathrm{~h}$ following formalin injection. Data collected between 0 and 10 min post-formalin injection compose phase 1, and data collected between 15 and 60 min post-iormalin injection represent phase 2 (Abbott et al., 1995).

\subsection{Volume changes of rat hind paw inflammation}

Paw edema was induced by injection of $100 \mathrm{~mL}$ of carrageenan (final concentration $15 \mathrm{mg} / \mathrm{kg}$ ) into the rat left hind paw. Edema was followed by measuring changes in paw volumes using a plethysmometer (Ugo Basile, Italy) at various times $(0,1,2,3,4$ and $5 \mathrm{~h})$. Before carrageenan treatment, the rats receive in the same paw an injection of vehicle, dexamethasone (100 $\mathrm{mL}, 1 \mathrm{~h}$ before) or Botaf (20 min beiore) at concentrations as indicated in the text. The increase in paw volume is considered as an index of inflammation intensity (Dỉ ROSA and Sorrentino, 1968). To measure paw volume fluctuation, the injected hind paw was immersed into a water cell filled with a solution of $\mathbf{0 . 0 4 e} 0.05 \%$ NaCl according to manuiacturer's instructions, and the volume of spillage considered as the volume of hind paw. 


\subsection{Analyses of c-ios/c-jun expression profiles}

Lumbar spinal cords were quickly removed by syringe aspiration after decapitation of mice ( $\mathrm{n} 1 / 43$ per group), $3 \mathrm{~h}$ after drugs or saline (control) administration and put immediately in nitrogen beiore mRNA extraction. Total RNA were prepared from mouse spinal cords using Macherey-Nagel Kit (NucleoSpin RNA II) and were converted in cDNA using First Strand cDNA Synthesis kit (GE Healthcare). Transcripts firom c-ios or c-jun were quantífied using TaqMan probes obtained from Applied Biosystems (Foster City, Ca). Calculations were periormed using mRNA c-ios or c-jun/mRNA glyceraldehyde 3 phosphate dehydrogenase (GAPDH) ratio. GAPDH was chosen as a house keeping gene.

\subsection{Electrophysiological study}

Adult rat DRG neurons were isolated and cultured according to the method described by Herzog et al. (2003). Briefly, rats were anesthetized and decapitated. Cells were treated with collagenase (1 $\mathrm{mg} / \mathrm{mL})$ and papain $(1 \mathrm{mg} / \mathrm{mL})$, dissociated in DMEM supplemented with $10 \%$ fetal bovine serum, and plated on glass coverslips coated with poly-L-ornithine and laminin. Cultures were maintained at $37{ }^{\circ} \mathrm{C}$ in a $5 \% \mathrm{CO}_{2}$ incubator, and media was changed every 2 days during experimental incubation periods. DRG neurons express both tetrodotoxin-sensitive (TTX-S) and TTX-resistant (TTXR) sodium channels.

Whole-cell recordings were periormed on DRG neurons. Membrane currents were measured with pipettes (2e5 MU) pulled from glass capillary tubes and connected to an EPC-9 amplifier operating Pulse:Pulsefit software (HEKA elektronik, Germany). The pipette solution was composed of (in mM): CsCl 120, TEACl 20 , $\mathrm{Na}_{2} \mathrm{ATP} 5$, EGTA 10, HEPES 10, $\mathrm{MgCl}_{2} 2.5$, $\mathrm{Na}_{2} \mathrm{GTP} 0.4$. The pH of the solution was adjusted to 7.3 with CsOH. The external solution was composed of (in mM): NaCl 140, KCl 5, CaCl $_{2} 2.5$, MgCl $_{2} 1$, HEPES 10 , D-glucose 10. The solution was adjusted to $\mathrm{pH} \mathrm{7.3e7.4}$ with NaOH. Ag-Agcl saline bridge was used for the reference electrode. The peak sodium currents were selected out and normalized by their relative amplitude to control.

\subsection{Iodination of BotaF and synaptosome preparation}

BotaF was iodinated by the lactoperoxidase method of $\mathbf{I}^{125}$ I] iodide oxidation according to Rochat et al. (1970). The specífic radioactivity was found to be $200 \mathrm{Ci} / \mathrm{mmol}$. The native BotaF was tested for its ability to displace $\left[^{\mathbf{1 2 5}} \mathrm{I}\right]-$ BotAF from the binding site of synaptosome P2 preparations (Gray and Whittaker, 1962). Synaptosomes $(3.3 \mathrm{mg} / \mathrm{mL})$ were incubated in a medium containing $130 \mathrm{mM}$ choline chloride, $5.4 \mathrm{mM}$ KCl, $5.5 \mathrm{mM}$ glucose, $0.25 \%$ bovine serum albumin, 50 mM HEPES, pH 6.5; 0.2 nM [ $^{125}$ I]-BotAF and increasing concentrations of BotAF were then added. Aiter 30 min at $37^{\circ} \mathrm{C}$, the synaptosomes were centrifuged (11,000 $\mathrm{x}$ g for $1 \mathrm{~min})$, the residual pellets were washed twice with the same medium and the bound radioactivity measured. The values are means of duplicate determination.

\subsection{Efiect of BotAF on [ $\left.{ }^{3} \mathbf{H}\right]$-ryanodine binding to SR vesicles}

SR vesicles from rabbit muscles were prepared as described previously in Marty et al. (2000). For the [ $\left.{ }^{3} \mathrm{H}\right]$-ryanodine binding assay, heavy SR vesicles (1 mg/mL) were incubated at $37^{\circ} \mathrm{C}$ for $3 \mathrm{~h}$ in an assay bufier composed of $5 \mathrm{nM} \mathbf{~ I}^{3} \mathrm{HJ}$-ryanodine, $150 \mathrm{mM} \mathrm{NaCl}$, 2 mM EGTA, $2 \mathrm{mM} \mathrm{CaCl}_{2}$ (pCa $1 / 4$ 5), and $20 \mathrm{mM}$ HEPES, pH 7.4. BotAF was added to the assay buffer just prior the addition of heavy SR vesicles. [ [ $\left.{ }^{3} \mathrm{H}\right]$-ryanodine bound to heavy SR vesicles was measured by filtration through Whatmann GF/B glass filters followed by three washes with $5 \mathrm{~mL}$ of ice-cold washing bufier composed of $150 \mathrm{mM}$ NaCl, 20 mM HEPES, pH 7.4. Filters were then soaked overnight in $10 \mathrm{~mL}$ scintillation cocktail (Cybscint, ICN) and bound radioactivity determined by scintillation spectrometry.

\subsection{Statistical analyses}

Statistics and determination of $\mathbf{E D}_{50}$ values were done using GraphPad Prism ${ }^{\circledR}$ (version 4.02, GraphPad, San Diego, CA). The results are expressed as mean \pm S.E.M with the exception of the $\mathbf{E D}_{\mathbf{5 0}}$ value (the dose of compound that produces $50 \%$ of the efiect relative to the control value) value, which was reported as the geometric mean accompanied by respective $95 \%$ confidence limits. ED $_{50}$ values were determined by non-linear regression analysis using a sigmoid dose-response equation. Ratios were considered significant if the confidence interval did not overlap. $N$ 1/4 6 animals for each dose of compound used in all tests. The level of significance was set at $5 \%(P<.05)$, and denoted by one *

\section{Results}

\subsection{Purification of a new analgesic peptide firom Bot venom}

$120 \mathrm{~mL}$ of scorpion Bot venom was water extracted, centrifuged and the supernatant was fractionated using gel filtration chromatography on Sephadex G50 (Miranda et al., 1970). Five fractions were resolved: MI, MII, BotG50, III and IV (Fig. 1A). Fractions were lyophilized and resuspended in water at $0.3 \mathrm{mg} / \mathrm{mL}$. Toxicity assays were performed on mice by i.c.v. injection of $0.1 \mathrm{mg} / \mathrm{kg}$ or by i.p. administration of $5 \mathrm{mg} / \mathrm{kg}$ of each fraction. At this concentration, fractions MI, MII, III and IV were non-toxic in opposition to BotG50wich was lethal. These fractions were tested for their ability to induce analgesia in mice using the writhing test. Fractions were injected i.p. at $5 \mathrm{mg} / \mathrm{kg}$ and evaluated for analgesia $1 \mathrm{~h}$ later. As shown, only iraction MII displayed a significant decrease in abdominal writhes (Fig. 1B). Next, the MII fraction was further fractionated by reversed-phase (8 semi-preparative HPLC (Fig. 1C). Among the collected fractions, only fraction F10 i.p. administrated at $5 \mathrm{mg} / \mathrm{kg}$ (arrow in Fig. 1C) displayed analgesic effect using the writhing test (Fig. 1D). At this stage, two factors prevented further characterization of the active peptide: (i) a poorly resolved peak as determined by C8 HPLC and (ii) the fact that the F10 fraction is only a minor component of the MII fraction. To solve this issue, we next determined whether a fraction similar to F10 in terms of elution profile may be present also in the BotG50 iraction, which seemed possible because of the partial overlap in MII and BotG50 iractions (Fig. 1A). As shown by the HPLC profile of the BotG50 fraction, a fraction that presents similar characteristics to F10 is also present in the BotG50 iraction, but in greater amounts and with better resoIution (Fig. 1E). In BotG50 c8 chromatographic profile F10 appears as a pool of smaller fractions defined as FI, F28, FIII, FIV and FV, that were all further evaluated for their toxicity in mice. None of these smaller fractions were toxic up to $0.1 \mathrm{mg} / \mathrm{kg}$ by i.c.v. and $5 \mathrm{mg} / \mathrm{kg}$ by i.p. route which permitted their evaluation for the writhing test pain model. of all these fractions tested, only F28 has the ability to attenuate abdominal contortions in mice upon i.p. injection at $2 \mathrm{mg} / \mathrm{kg}$ (Fig. 1F). When further resolved on an analytical reversedphase c18 column, F28 eluates as a single well-resolved peak at a fixed retention time of 14 min encompassing a molecule that we termed BotaF for Buthus occitanus tunetanus Analgesic Factor (Fig. 1G). In this elution profile, other minor contaminants were also present. Intraperitoneal pretreatment of mice with $2 \mathrm{mg} / \mathrm{kg}$ of purífied BotaF was sulficient to attenuate up to $85 \%$ of abdominal cramps, whereas injection, in same experimental conditions, of a collected pool of the contaminants, had no effect (Fig. 1H). 
A.

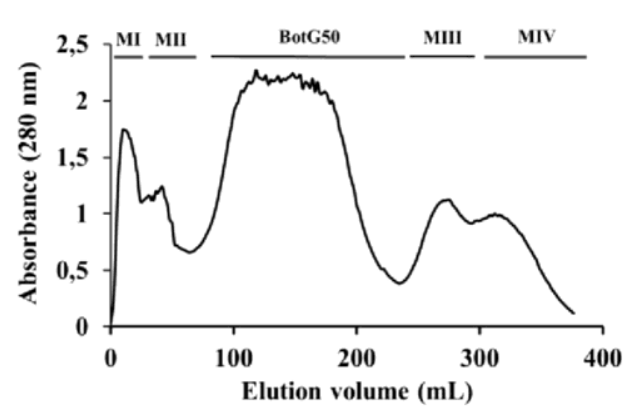

D.

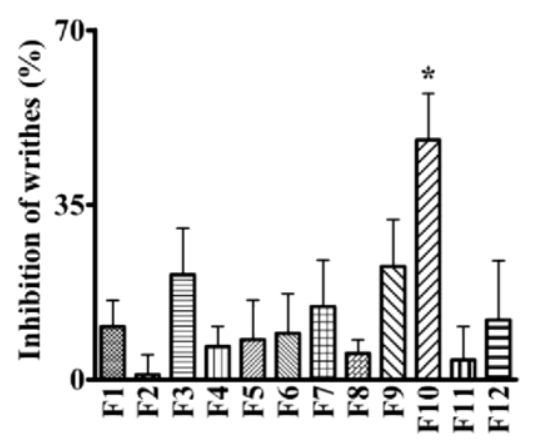

Treatment $(5 \mathrm{mg} / \mathrm{kg})$

G.

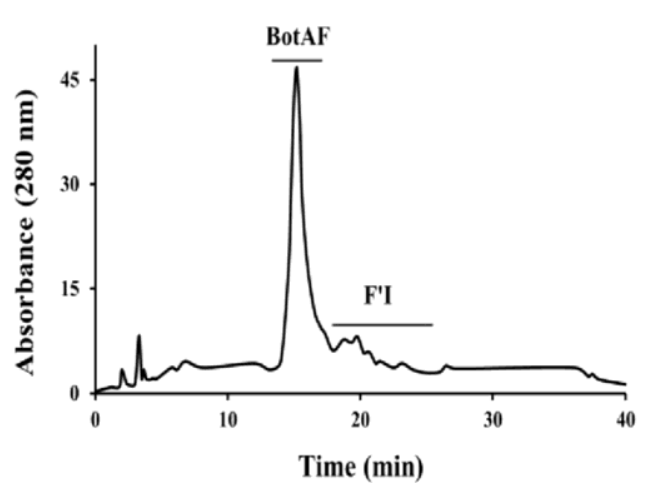

B.

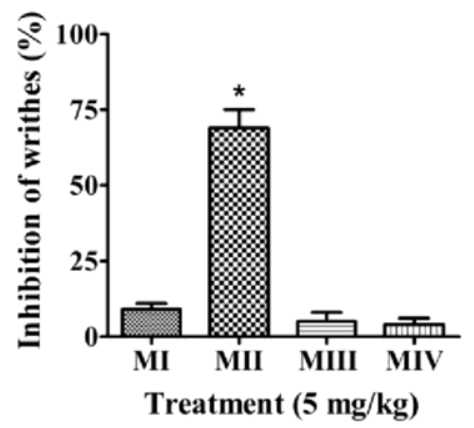

E.

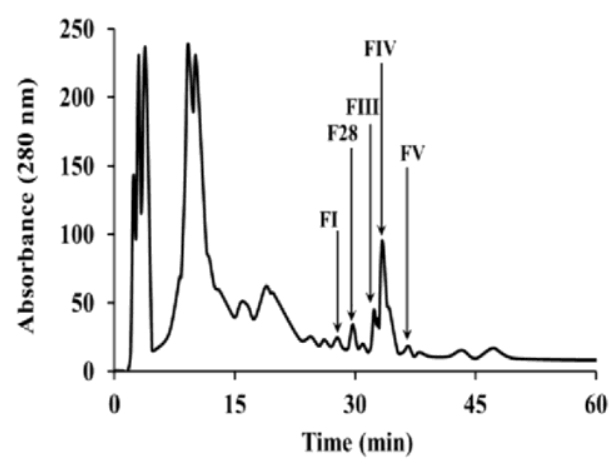

H.

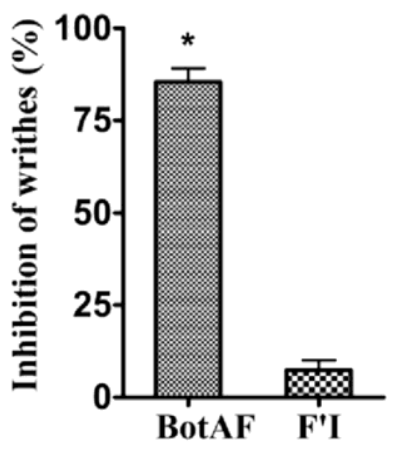

Treatment $(2 \mathrm{mg} / \mathrm{kg})$
C.

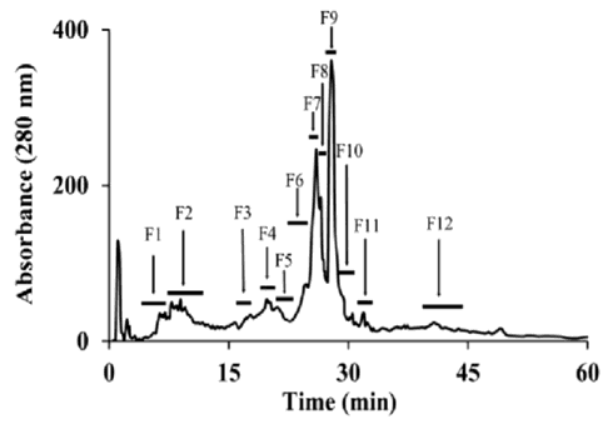

F.

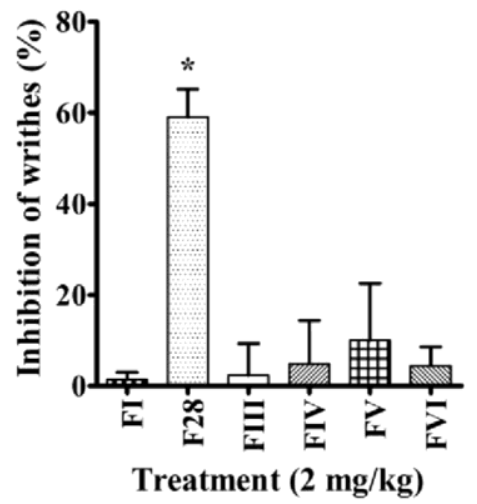

I.

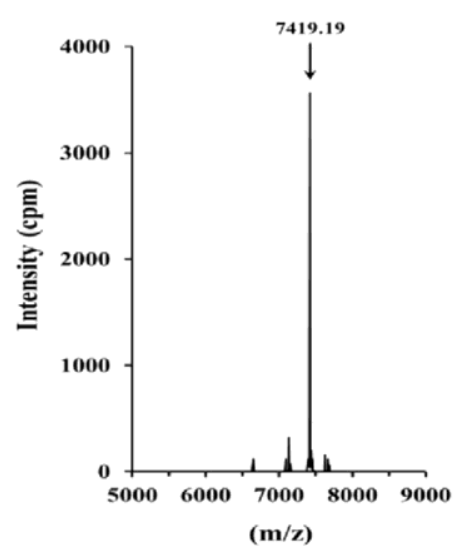

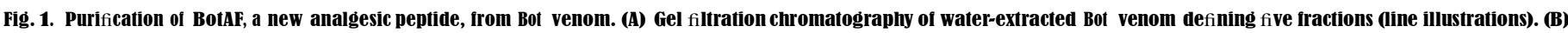

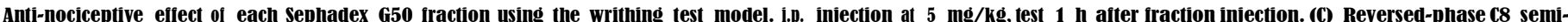

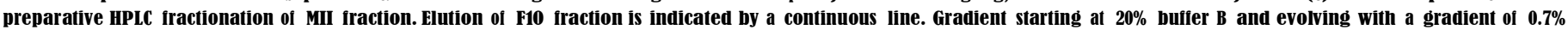

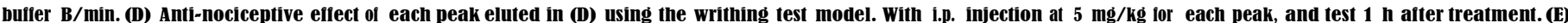

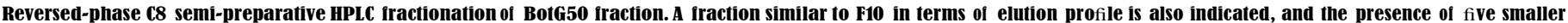

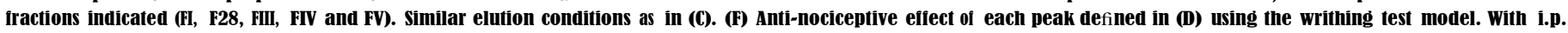

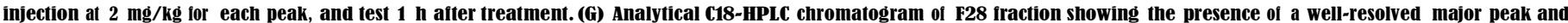

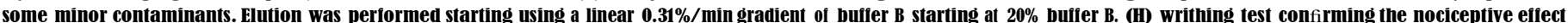

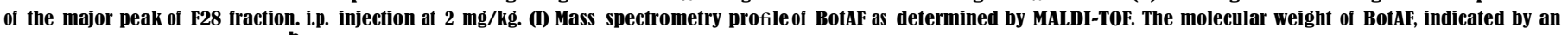
arrow, correspond to the (MpH) ${ }^{\mathbf{b}}$ ion.

According to crude estimations, Botaf represents less than $1 \%$ of peptide components of the BotG50 iraction and less than $0.5 \%$ of total venom extract. BotAF has an experimental mass (MpH ${ }^{\mathbf{b}}$ ) of 7419.19 Da as determined by MALDI-TOF mass spectrometry (Fig. 1I). This result indicates that BotaF belongs to a long-chain class of scorpion toxins. Amino acid analyses indicate that BotAF is pure at $98 \%$. 
A.

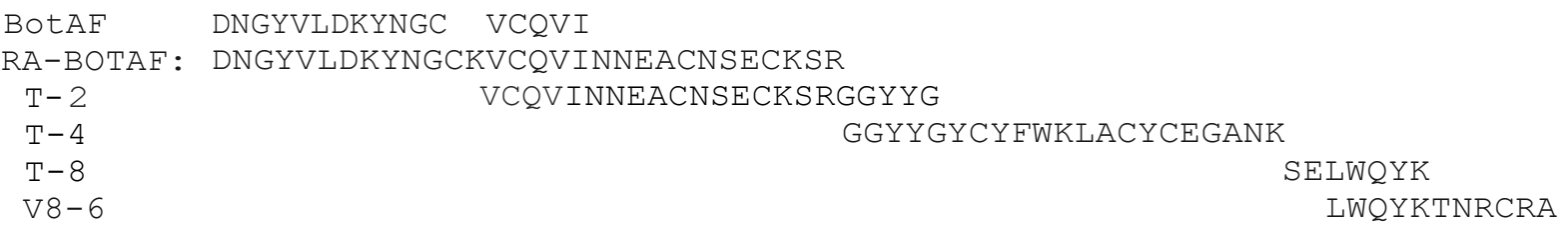

B.
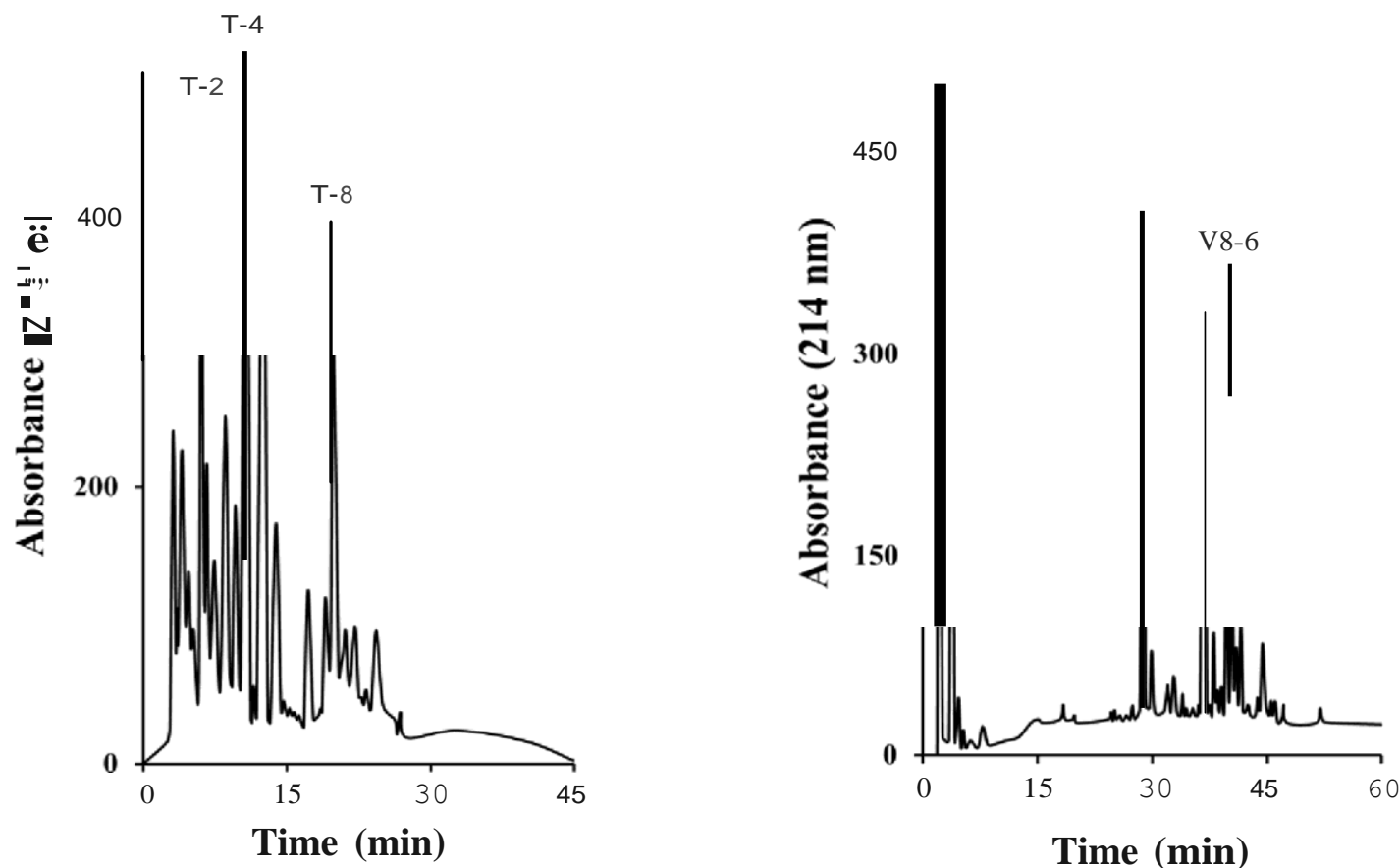

Time (min)

C.

1

10

20

30

40

50

GAT AAT GGT TAC GTA CTT GAC AAA TAT AAC GGT TGC AAG GTC TGT CAG GTT ATT AAA

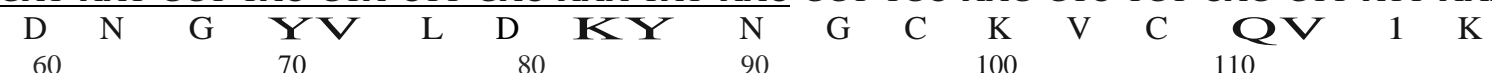

AAT GAA GCT TGT GAT AGT GAG TGT AAA TCA AGA GGT GGA ACT TAC GGC TAC TGC TAC

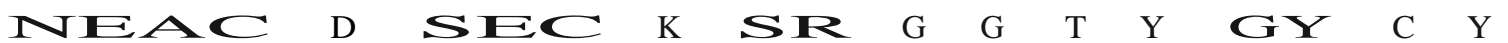
120

$130 \quad 140$

$160 \quad 170$

TTC TGG AAG TTG GCC TGT TAT TGC GAA GGT GCT AAC AAA TCA GAG CTT TGG CAA TAC

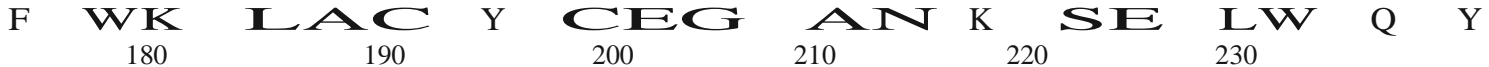

AAA ACA AAT AGA TGC CGT GCA TGA ATATGCACTGCATCGATTTGCATGAATATTTATAATA

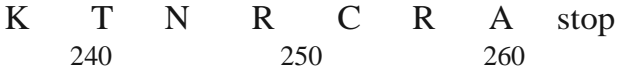
270
280
290
300

TTCATGCAAATAGATGCATGAATATTATAAATTCAATATTTAATTTCTGTACGGTTCAAATATAT

$\begin{array}{rrrrr}310 & 320 & 330 & 340 & 350\end{array}$

AACTGAAACTAAAATATATTAAAGTATTATCGATTTATATGTAAAGCATATGTAAaataaaTAT AT

370

380

390

400

410

TTTGGCATGGCAAAAAAAAAAAAAAAAAAAGTACTAGTCGACGCGTGGCC

D. 


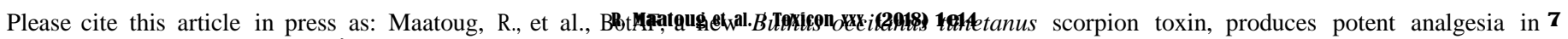
rodents, Toxicon (2018), https:ffdoi.org/10.1016/j.toxicon.2018.01.003 



\subsection{Amino acid sequence of Botaf and sequence homology}

To determine the amino acid sequence of BotAF, $5 \mathrm{nmol}$ of peptide was first reduced by $1.4 \mathrm{mmol}$ DTT. The reduced peptide was then alkylated with 4-VP (to yield reduced-alkylated BotAF (RABotAF), and subjected to direct Edman degradation. A partial sequence was obtained from the $\mathrm{N}$-terminus up to amino acid position 30 (Fig. 2A). To get additional sequence data, RA-BotAF was also separated by SDS-PAGE and gel-digested with either trypsin or S. aureus v8 protease, as described in Materials \& Methods. The proteolytic peptide fragments from both digests were extracted from gel slices and separated by C18 analytic HPLC (Fig. 2B). As shown, trypsin digestion results into at least 14 major peptide fragments (leit panel), whereas v8 protease digest yielded at least 13 iragments (right panel). Fragments T-2, T-4 and T-8 from trypsin digest and fragment v8-6 from v8 protease digest were purified and sequenced by Edman degradation. The resulting sequences are provided in Fig. 2A. Sequence overlapping analysis of the $\mathbf{N}$-term sequence of RA-BotAF, T-4 and T-2 was used to determine the correct order in the final sequence. T-8 was 7 residues long and seems to prolong $\mathrm{T}-4$ sequence to the C-terminus according to sequence homology with BmKaS and BmKas1 (Fig. 3). The v8-6 iragment overlaps and prolongs the T-8 sequence and should complete the sequence of Botaf according to the molecular mass obtained. In fact the theoretical mass of reduced BotaF is 7427.37 Da, which should correspond to a theoretical mass of 7419.37 ior folded/oxidized BotAF, a value identical to the experimental mass obtained. BotAF is therefore a peptide of 64 amino acids, presumably containing 4 disulfide bridges.

To search for BotaF-like sequences, we therefore undertook the cloning and sequencing of cDNAs coding for Botaf-like toxins. The mRNA was extracted from Bot venomous glands and CDNA was synthesized by reverse transcription. Resulting cDNAs were PCRamplified with a couple of predesigned primers: a forward primer designed on the basis of the $\mathrm{N}$-terminal amino acid sequence of BotaF and the cDNA sequence of three homologous toxins, BmKas, BmKas1 and BotIT2, and a reverse olígo dT sequence. Amplífied PCR products were subcloned into pGEM-T vector and sequenced. Several cDNA sequences were obtained coding for b-like long-chain toxins, but one sequence matched more closely what could be expected for the cDNA sequence of BotAF (Fig. 2C). It encodes the Botaf2 pro-toxin. The open reading frame for the BotaF2 pro-toxin is 196 bps-long ending with a TGA stop codon. A polyadenylation signal is located $157 \mathrm{bps}$ from the termination codon. Translation of this sequence yields BotAF2 (Fig. 2D), which differs irom BotaF by three amino acids, namely $\mathrm{Lys}^{19}$ instead of $\mathrm{Asn}^{19}$, $\mathrm{Asp}^{24}$ instead of $\mathrm{Asn}^{24}$, and $\mathrm{Thr}^{33}$ instead of Tyr $^{33}$. BotAF2 also contains 64 amino acids and should fold according to the same pattern as BotAF. The predicted molecular mass of BotAF2 is 7391.33.

\subsection{BotAF and BotAF2 are two new members of the b-like long- chain scorpion toxins}

A blast search was periormed to identify toxins sharing sequence homology with BotAF and BotAF2 sequences. Interestingly, there is a high homology (>70\%) with b-like scorpion toxins (BmKAS, BmKAS-1, BmP09, Lqhb1 and AaHIT4). Multi sequence alignment for BotAF using Clustalw software was periormed (Fig. 3A.). Sequence identities ranged from 70\% (AaHIT4) to $\mathbf{7 8 \%}$ (BmKAS). Clustalw alignments were also periormed between BotAF and depressant anti-insect toxins with characterized analgesic activity (except for BotIT2), and results presented in Fig. 3B show that BotaF is sharing also high identity with this group, albeit lower (between 43 and 50\%).

\subsection{Toxicity and rotarod tests}

No mortalíty was noticed $24 \mathrm{~h}$ after treatment of mice by increasing doses of BotAF by i.c.v. route (up to $0.5 \mathrm{mmol} / \mathrm{kg}$ ) or by i.p. route (up to $1 \mathrm{mmol} / \mathrm{kg}$ ). Since no external signs of toxicity were observed, we conclude that Botaf exhibits no toxicity in vivo (see Materials \& Methods section).

The Rotarod experiments demonstrate that BotAF at antinociceptive doses has no efiect on motor function. In fact, as shown in Fig. 4A, no significant difference is seen between the drop latency time in mice groups treated with BotAF (0.5, $1 \mathrm{mmol} / \mathrm{kg})$ i.p. or $(0.2,0.5 \mathrm{mmol} / \mathrm{kg})$ i.t. versus control groups (treated with vehicle in the same conditions).

\subsection{Anti-nociceptive effect of BotaF in writhing and hot-plate tests}

Using the anti-nociceptive writhing test, BotaF has no efiect when injected by i.c.v. or i.v. routes up to $0.3 \mathrm{mg} / \mathrm{kg}$ (data not shown). In contrast, a significant reduction in acetic acid-induced contortions was observed if BotaF is administered by i.p. route (Fig. 4B). This effect is dose-dependent and reaches maximum at $5 \mathrm{mg} / \mathrm{kg}$. Optimal anti-nociception is observed if BotaF is injected i.p. 60 min beiore the test, but good anti-nociception (up to $50 \%$ ) is still observed 90 min after BotaF administration. A dose-response curve was constructed and compared to the effect of b-endorphine (Fig. 4C). The $\mathbf{E D}_{50}$ of BotAF is $154.2 \mathrm{mmol} / \mathrm{kg}$ (i.p., $60 \mathrm{~min}$ before test), while that of b-endorphine is $358.4 \mathrm{nmol} / \mathrm{kg}$ (i.p., 40 min before test). These results indicate that BotAF is a 2.3 -iold more potent anti-nociceptive compound than b-endorphine for viscero-somatic pain by i.p. administration.

Next, we evaluated the efficacy of Botaf on preventing acute somatic nociception by using the hot-plate test (Fig. 4D). BotAF dose-dependently attenuates somatic pain by prolonging the paw withdrawal latency of C57/BL6 mice to hot plate stimuli. This antinociceptive activity is dependent on the time between the treatment and the nociceptive activity test. The maximum effect occurs 60 min aiter i.p. injection. In comparison, b-endorphine reaches maximal effect 30 min after i.p. injection, but the effect is prolonged for more than $1 \mathrm{~h}$ after this peak. Dose-response curves illustrate an ED $_{50}$ of $788.4 \mathrm{nmol} / \mathrm{kg}$ (i.p., $60 \mathrm{~min}$ before test) for BotAF, while that of b-endorphine is $1995 \mathrm{nmol} / \mathrm{kg}$ (i.p., $40 \mathrm{~min}$ beiore test). Here, again BotAF is 2.5-iold more efficient than b-endorphine upon i.p. injection. In this test also Botaf has no effect by i.c.v. or i.v. injections.

\subsection{Effect of Botaf on tail-flick test behavior}

The i.p. injection of BotaF significantly and dose-dependently increases the tail withdrawal latency (Fig. 5A). The maximal antinociceptive effect is obtained, like in the hot-plate test, at $60 \mathrm{~min}$ aiter treatment and anti-nociception is still significant up to 120 min aiter peptide injection. Evaluation of dose-response curves provides ED Fo $_{51}$ 517.3 $\mathrm{nmol} / \mathrm{kg}$ for BotAF (i.p., $60 \mathrm{~min}$ before test), while that of b-endorphine is $2401 \mathrm{nmol} / \mathrm{kg}$ (i.p., $40 \mathrm{~min}$ beiore test) (Fig. 5B). BotaF remains thereiore more potent than bendorphine, with about 4-iold difierence this time. These data indicate that there are not many difierences in efficacy of BotAF with regard to the somatic pain challenged if injected i.p.

The analgesic activity was further investigated in this model by i.t. injection. Again, a maximal efiect of BotaF on tail withdrawal latency was observed 60 min aiter its i.t. injection (Fig. 5c). This efiect occurred at lower concentrations than upon i.p. injections (Fig. 5C and D), and was more transient in duration. Evaluation of the dose-dependence of this effect yields an $\mathbf{E D}_{50}$ of $\mathbf{5 7 . 3 8} \mathrm{hmol} / \mathrm{kg}$ (60 min before test), which is thus about 9-fold more potent than 
A: $\beta$-like toxin

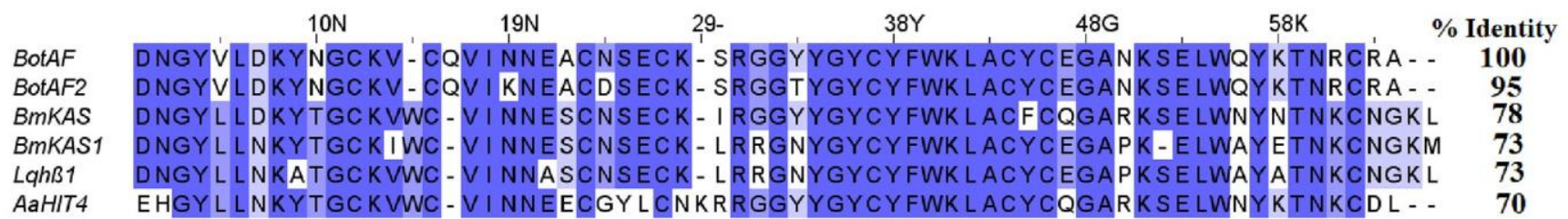

B: Depressant toxins (anti-insect)

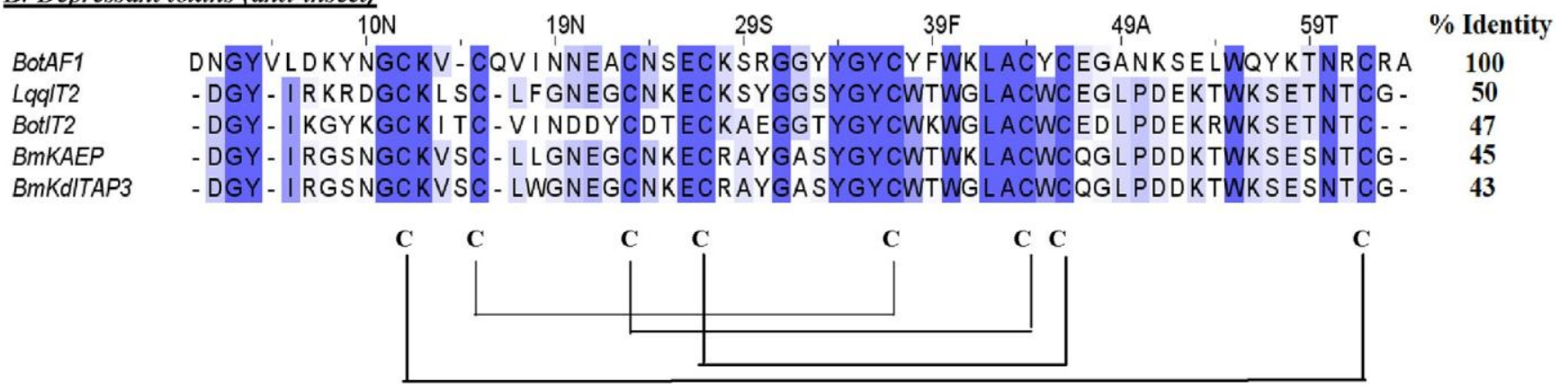

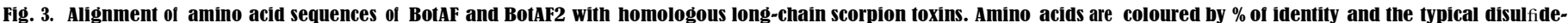

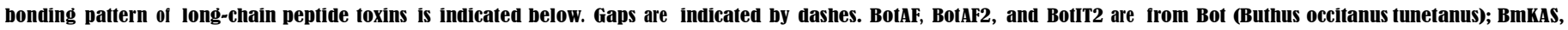

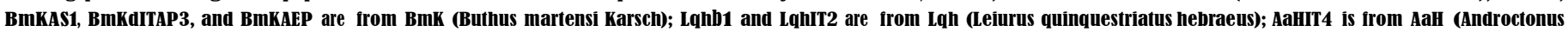
australis hector); LqqIT2 is from Lqq (Leiurus quinquestriatus quinquestriatus).

by i.p. administration (Fig. 5D).

\subsection{BotAF antinociceptive effect by i.pl. injection in the formalin} test

The intraplantar injection of $2.5 \%$ formalin into the left hind paw produces a consistent flinch and lifiting/licking response in rat. The time course curves of these counted behaviors is biphasic in time (Fig. 6A), comprising two phases: phase 1, lasting 0 e5 min and corresponding to acute inflammatory pain and phase 2, lasting 10 e60 min and corresponding to chronic inflammatory pain. As shown in Fig. 6A, BotaF (0.23 $\mathrm{mmol} / \mathrm{kg}$ or $0.47 \mathrm{mmol} / \mathrm{kg})$ intraplantary injected 30 min beiore formalin, significantly attenuates pain behaviors of both phases 1 and 2 as compared to control group. Average flinching scores of BotAF during phase 1 and phase 2 are shown in Fig. 6A and compared to the scores obtained for profenid (12 $\mathrm{mmol} / \mathrm{kg}$, i.pl. $30 \mathrm{~min}$ beiore test) and for morphine sulfate (0.6 $\mathrm{mmol} / \mathrm{kg}$, i.pl. $30 \mathrm{~min}$ before test). As expected, profenid produces significant anti-nociception only on the inflammatory phase, while morphine sulfate is active on both phases.

Dose-response curves of all three compounds, administrated by the intraplantar root (i.pl.), are shown on both phases except for profenid (Fig. 6B). As shown, the $\mathbf{E D}_{50}$ for BotAF are $225.7 \mathrm{nmol} / \mathrm{kg}$ (phase 1) and $204.9 \mathrm{nmol} / \mathrm{kg}$ (phase 2), while $\mathbf{E D}_{50}$ for morphine sulfate are $552.2 \mathrm{nmol} / \mathrm{kg}$ (phase 1) and $442.9 \mathrm{nmol} / \mathrm{kg}$ (phase 2). These data suggest that BotAF, injected locally, acts as well on phase 1 than on phase 2 , similarly to morphine suliate. On average, potency ratios show that BotaF is more efficient by 2 fold than morphine sulfate.

\subsection{Modulation of $\mathrm{c}$-ios/c-jun expression by BotaF in visceral and somatic pain conditions}

As shown, in Fig. 7, the i.p. injection of acetic acid, produces a drastic increase in c-ios and c-jun mRNA levels $2 \mathrm{~h}$ after viscerosomatic pain induction. The i.t. administration of Botaf dosedependently inhibits this mRNA expression increase. This inhibition is complete for c-ios and c-jun. Similarly, using a somatic inflammatory pain model (i.pl. formalin injection $2 \mathrm{~h}$ before assessing mRNA levels), BotAF pretreatment (i.p., 15 min before formalin injection) dose-dependently prevents c-ios and c-jun mRNA up-regulations (Fig. 7C and D). In this model also, the inhibition of mRNA level increase is complete for both c-ios and c-jun. Furthermore result in Fig. $7 \mathrm{C}$ and D shows that BotAF $(0.4 \mathrm{mmol} / \mathrm{kg}$, i.p.) when injected alone (with no other drug) does not induce any significant up-regulation of c-fos and c-jun mRNA level as compared to non-treated control group.

\subsection{Pharmacology of BotAF anti-nociceptive activity}

The pharmacological activity of BotaF was investigated on voltage-gated $\mathrm{Na}^{\mathrm{b}}$ currents in rat DRG neurons maintained in primary cultures. The membrane potential of rat DRG neurons was held at $\mathbf{- 8 0} \mathrm{mV}$, which was near the resting membrane potential. Whole-cell path-clamp recording found that about only $10 \%$ of peak total sodium currents of rat DRG neurons could be significantly inhibited by $1 \mathrm{mM}$ of BotaF (Fig. 8A). This inhibition could be largely recovered upon washout of Botaf. We also assessed the effect of $1 \mathrm{mM}$ BotaF on the iraction of TTX-resistant currents and the results show that BotAF significantly inhibited (about 20\%) TTX-R currents (data not shown). It is thereiore concluded that BotAF is not a good $\mathrm{Na}^{\mathbf{b}}$ channel blocker and that its anti-nociceptive efiect is unlikely to involve block of $\mathrm{Na}^{\mathrm{b}}$ channels.

To measure the interactions of BotAF with CNS ion channels, competition binding assays to rat brain synaptosomes were performed with a fixed concentration of ${ }^{125}$ II-BotaF and increasing concentrations of Botaf. The results show that BotAF, with and $\mathbf{I C}_{50}$ of $2.2 \mathrm{mM}$, has a low aifinity for rat brain synaptosomes receptors (Fig. 8B). Similarly, BotaF activity was tested on ryanodine receptors. As shown, $1 \mathrm{mM}$ Botaf has no effect on $\left.I^{3} \mathrm{H}\right]$-ryanodine binding onto SR vesicles (Fig. 8 C), indicating that ryanodine receptors are also not good targets for BotAF.

The involvement of the opioid endogenous system in the analgesic activity of BotaF was also assessed in the tail filck model. We 
A.

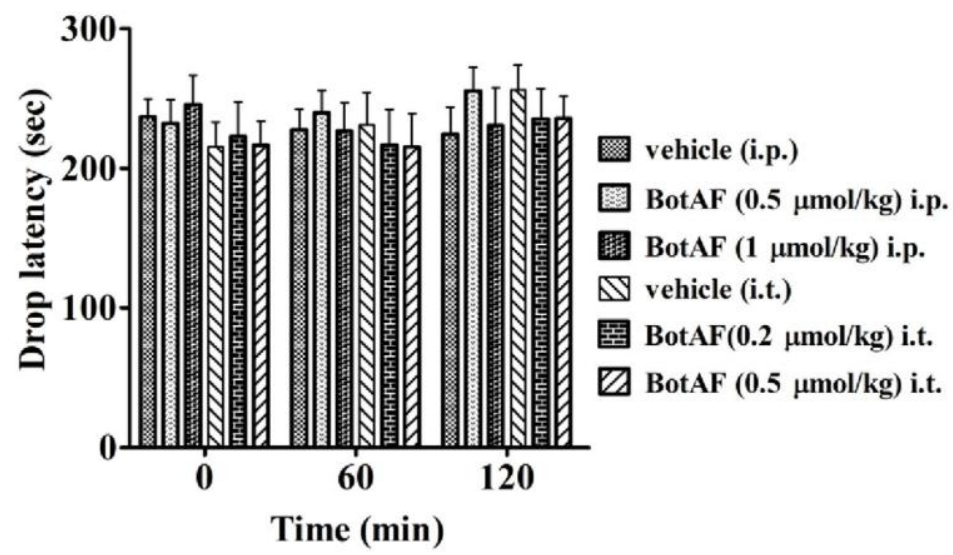

B.

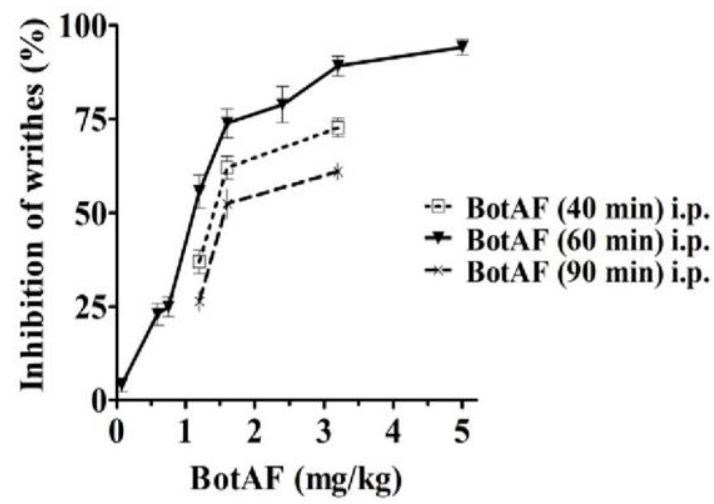

D.

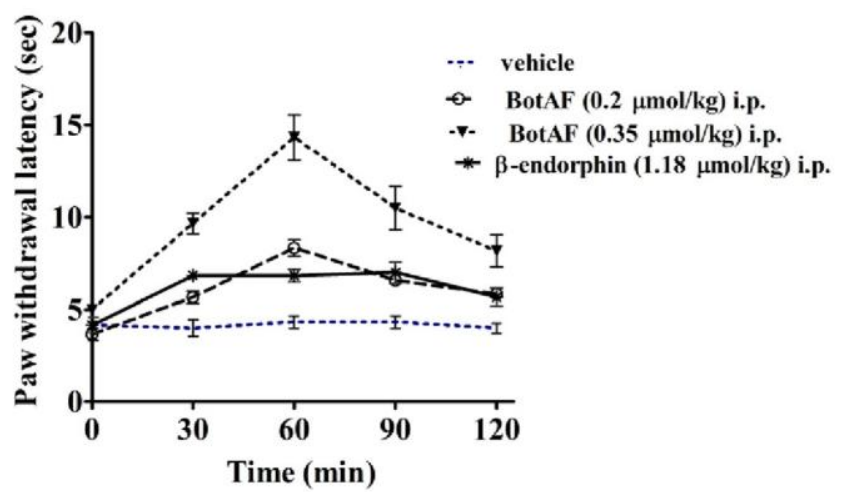

C.



E.

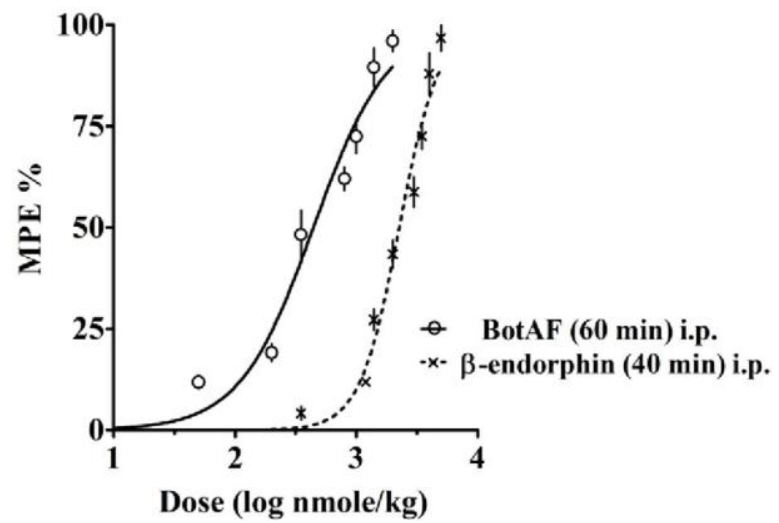

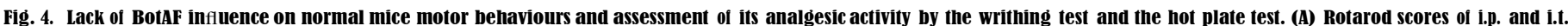

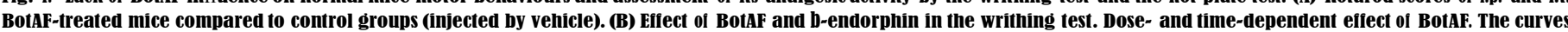

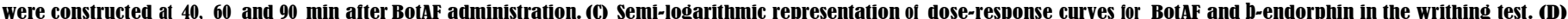

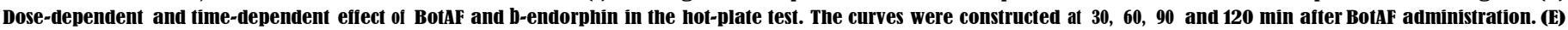
Semi-logarithmic representation of dose-response curves for BotaF and b-endorphin in the hot-plate test.

studied its possible attenuation by the opioid antagonist naloxone. As shown in Fig. 9A, naloxone pre-treatment does not afiect the anti-nociceptive activity of BotAF, assessed in the tail flick model, indicating that this peptide does not act on opioid receptors for its analgesic effect.

Finally, the anti-inflammatory activity of BotAF was determined in vivo by paw edema volume assessment. Dexamethasone (a wellknown AINS drug) or BotaF were injected intraplantary $1 \mathrm{~h}$ beiore paw edema induction by carrageenan injection. As shown in Fig. 9B, only dexamethasone potently inhibited paw inflammation, while BotAF injected intraplantary, up to $1.8 \mathrm{mmol} / \mathrm{kg}$, was inefiective in reducing Paw edema volume. 
A.

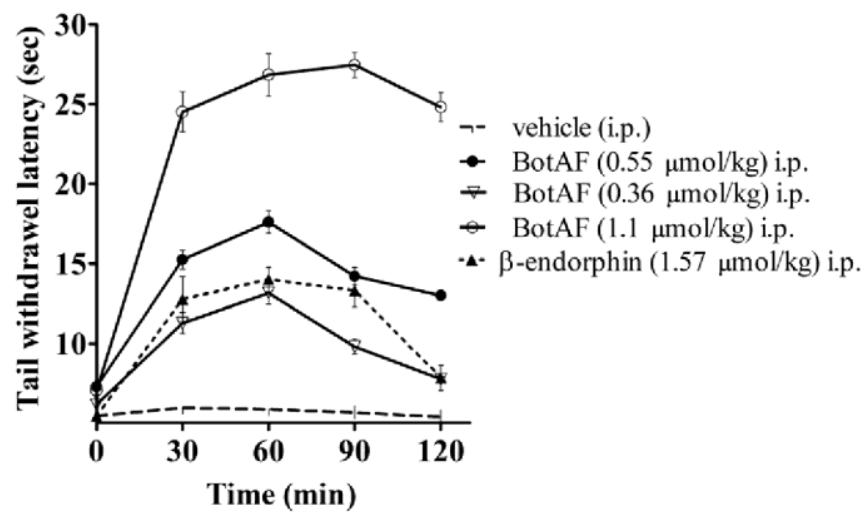

C.

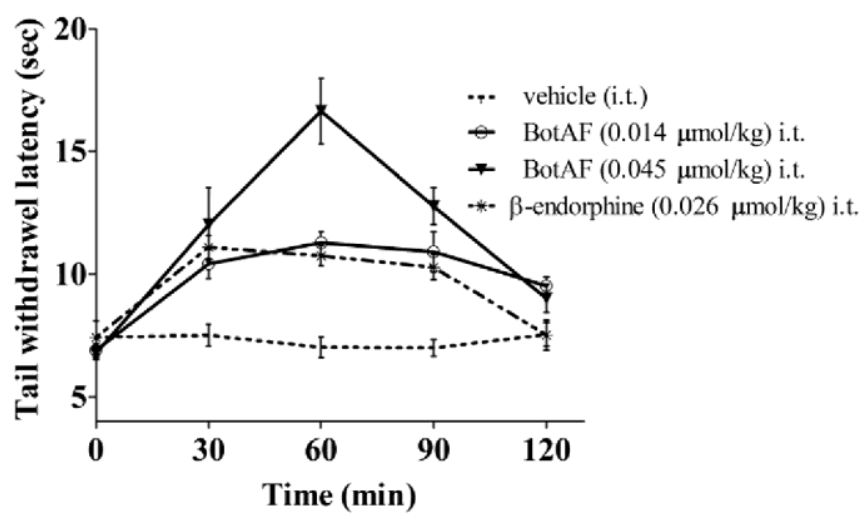

B.

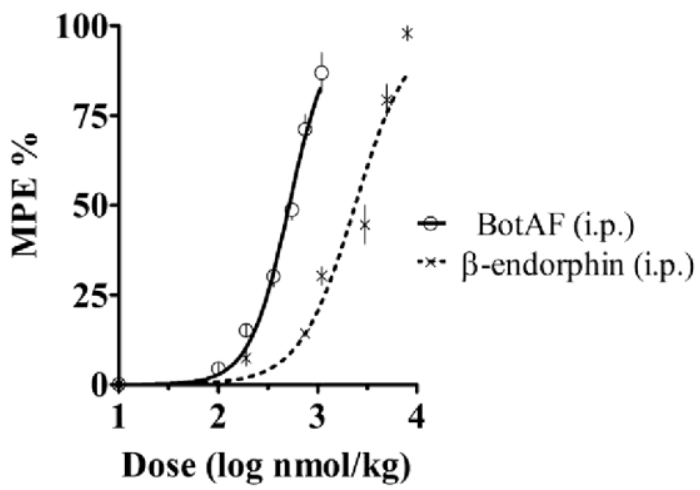

D.

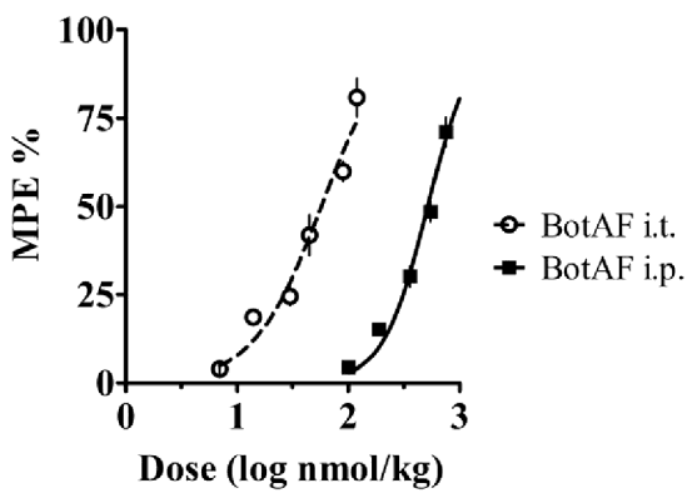

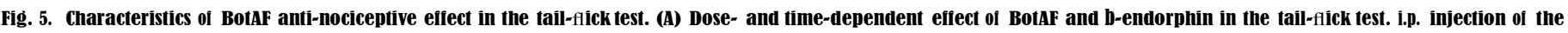

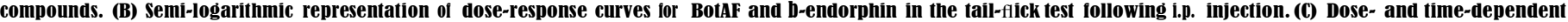

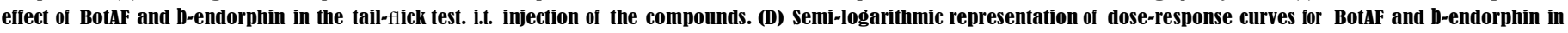
the tail-flick test following i.t. injection.

\section{Díscussion}

Here, we identífied a novel toxin, Botaf, that produces potent analgesic efiects in rodents. Botaf was purified from the venom of Bot using an activity-guided screening strategy. This consisted in isolating a compound from Bot venom fractions by assessing its anti-nociceptive effect in vivo together with confirming the lack of toxicity of the active compound. This strategy requires several conditions to be successiul: (i) an important quantity of venom provided by unique facilities, (ii) a non-toxic peptide, (ii) a highly active or sulficiently abundant compound and, (iii) a reliable (or several) pain model(s). In this chosen screening approach, the writhing test appears adequate since it is highly sensitive test that lacks selectivity allowing the identification of a larger number of molecules regardless of the pharmacological target involved in nociception. In addition, to satisfy the exigence of the screening approach, we deliberately focused on the G-50 venom fractions that are devoid of lethality upon i.c.v. or i.p. injection into mice. As most scorpion venom compounds are toxic, this approach should promote the discovery of original new active compounds. In agreement with this expectation, we indeed found BotaF to represent a minor compound with less than $1 \%$ of the venom content. This finding also illustrates to which extent the in vivo screening is valid.
The Botaf sequence was determined by direct sequencing. The whole sequence of Botaf consist of 63 amino-acids and the peptide has a MW of about 7419 Da. Homology alígnments demonstrate that BotaF structurally belongs to a family of recently characterized toxins, termed b-like scorpion toxins (Gordon et al., 2003). This family contains so far four members, all from the Buthidae family of scorpions: BmKAS, BmKAS1 (Lan et al., 1999), AaHIT4 (Loret et al., 1994) and Lqhb1 (Gordon et al., 2003). These toxins have a minimum of $70 \%$ sequence identity and vary in length from 63 to 66 amino acids. The consensus pattern for disulfide bridges would be $\operatorname{Cys}^{1}-\mathrm{Cys}^{8}{ }^{8}$ Cys $^{2}-\mathrm{Cys}^{5}, \mathrm{Cys}^{3}-\mathrm{Cys}^{6}$ and $\mathrm{Cys}^{4}-\mathrm{Cys}^{7}$. of relevance to our findings, two members of this family of toxins, BmKas and BmKAS1, have been reported as efficient analgesics by several tests (Chen and Ji, 2002; Chen et al., 2006; Shao et al., 2008). BotAF has also a level of sequence identity (between 43 and 50\%) with antiinsect depressant toxins such as BmKAEP (an analgesic and antiepileptic toxin). Several other members of the anti-insect depressant toxin family have analgesic properties as well (Guan et al., 2001; Bai et al., 2007). We reveal also very limited sequence identities with members of the b-toxin family, known for their toxicity in vivo. Beside its analgesic potential, the so-called beta-like toxin group shows several original features compared to other scorpions groups as it is composed by a restricted set of members sharing high sequence similarities but also peculiar and diverse 
A.

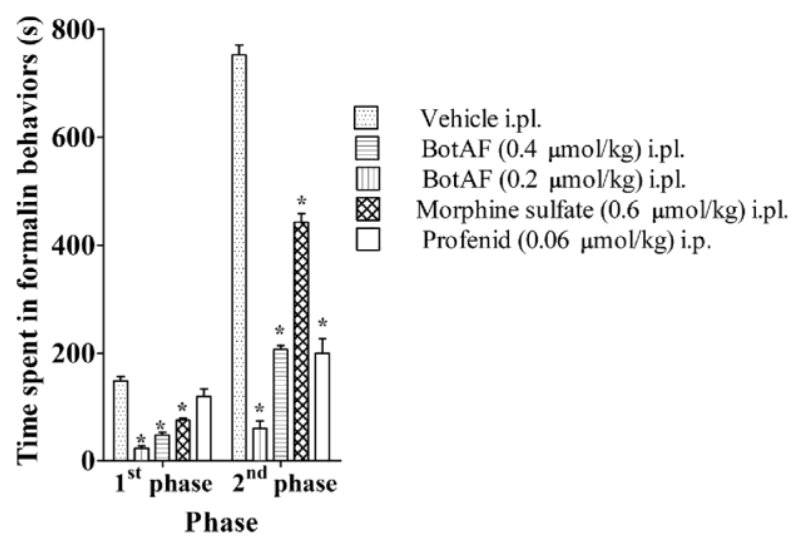

B.

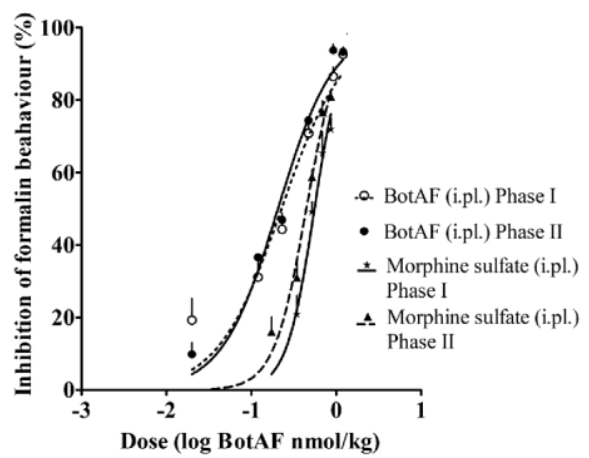

Fig. 6. Anti-nociceptive effect of BotaF in the formalin test. (A) Efiect of Botaf on averaged flinching scores for each phase: acute phase (phase 1) and an inflammatory phase (phase 2), compared to the effects of saline, profenid and morphine sulfate. (B) Dose-response curves of all tested compounds on both phases, except for profenid that was evaluated on phase 2 exclusively.

pharmacological profiles. Thus, Botaf constitutes by itself a very promising lead for future structure-function studies and for understanding the evolution of scorpion's toxins.

As Botaf seems to have a promising pharmacological profile, it seemed of interest to search for analogues within the same venom as alternative splicing of genes coding for toxins is a frequently used strategy to enrich the repertoire of active compounds. The success of this approach was exemplified herein by molecular biology as toxin cloning from Bot venomous glands provided the sequence of a new BotaF isoform, termed herein BotAF2. BotaF2 differs from BotaF by only three amino acid residues and is thereiore likely to possess the same anti-nociceptive efiect than BotaF itself. Nevertheless, this assumption can be proven true only when the full synthesis of Botaf2 will be periormed chemically or through recombinant means. For the time being, we did not find out in which fraction of the venom Botaf2 was present, possibly also because it also represents a minor component of the venom.

Our work also attempted to deepen the in vivo activity of BotAF. Toxicity of the molecule was investigated by means of lethality assessment after $48 \mathrm{~h}$ and by direct observation of any mice behavioral change that could refiect a form of intoxication summarized in bibliographic data in which these symptoms were well characterized (0zkan et al., 2007; Pipelzadeh et al., 2015). Besídes toxicity, it was also important to assess animal motor response in
BotaF administration condition. Many pain models used for investigating nociception in conscious laboratory animals rely upon an observable motor response and the analgesic potential is evaluated on the basis of changes in the latency of motor responses following nociceptive stimulation (heat, acetic acid...). Interpretation of an increase in response latency as evidence that analgesia takes place is invalid if the putative analgesic factor interferes with motor function (Warren and LSon, 1982). Such coniounding motor effects can be assessed in many ways, including the rotarod test. Thus, we used this particular test to measure whether or not BotaF affects the motor coordination of rodents over the time periods and experimental conditions used in revealing its antinociceptive activity. Using this procedure, we confirmed that behavioral changes in mice challenged with BotaF was not due to impairment of the normal motor function but to its anti-nociceptive action.

Next, the potency and specificity of the analgesic activity of BotAF was analyzed, first in vivo by assessment of animal behavioral changes in several pain models, and, secondly, in vitro by assessing nociceptors activation. We started with acute pain models: writhing test (model designed for visceral pain), hot plate test and tail fick test (somatic pain). In those models, Botaf shows a potent preemptive analgesia (with an optimal activity obtained when injected $1 \mathrm{~h}$ before pain induction) as confirmed by $\mathbf{E D}_{50}$ values compared to b-endorphine as a reierence analgesic drug. In addition, BotaF analgesic activity was further investigated on an inflammatory pain model: the formalin test. The data demonstrate that BotAF is efficient towards abolishing inflammatory pain with high potency compared to profenid and morphine-sulfate. The interesting potent analgesia of BotaF in all tested models is particularly informative when considering the different pain syndrome that they represent. In fact, such lack of specificity for a particular pain type (visceral/somatic/acute/inflammatory...) is in favor of an action of Botaf on the nerve conduction and/or the central transmission of pain information rather than on the pain induction processes themselves (stimulus transduction). In this context, it is worth reminding that the analgesic efficacy of other reported venom peptides is dependent on their mode of administration. This, in turn, has often limited their clinical use (see for instance Prialt restricted to i.t. injection (Rauck et al., 2009)), or restrained their usage to a preclinical level. Concerning BotAF, it was inefiective by the i.c.v. or i.v. roots. Conversely, the compound was highly effective when administrated by the i.t. root regardless of the pain etiology. It should therefore face similar challenges for the clinical applications as Prial. However, contrary to the later, BotaF shows a gain in potency in the formalin test when injected intrapodally which should facilitate to some extent its clinical application.

The analgesic activity of Botaf was further evaluated in vitro by quantifying medullar c-ios and c-jun mRNA expression. Painful visceral (acetic acid in peritonea) or somatic stimuli (intrapodal formalin injection) leads to a rapid and transient induction of the expression of the immediate early genes c-ios and c-jun in lumbar spinal cord (Barr et al., 2003; Bianchi et al., 2003). The level of expression of these immediate early genes is refiective of the spinal activation by the nociceptive input and is used therefore as an indirect marker of pain (Morgan and Curran, 1991). As expected, BotAF attenuates in a dose-dependent manner the induction of pain-related expression of both c-ios and c-jun. These observations were previously also reported for other analgesic scorpion toxins (Bai et al., 2007; Liu et al., 2008; Martin-Eauclaire et al., 2010). Also, the amount of Fos/Jun mRNA expression inhibition is correlated with the potency of analgesia. Several studies have shown that analgesics, given preemptively, reduce the extent of Fos expression in a dose-dependent manner with a good correlation with their potency (Abbadie et al., 1994; Abbadie and Besson, 1994). Hence, 
A.1

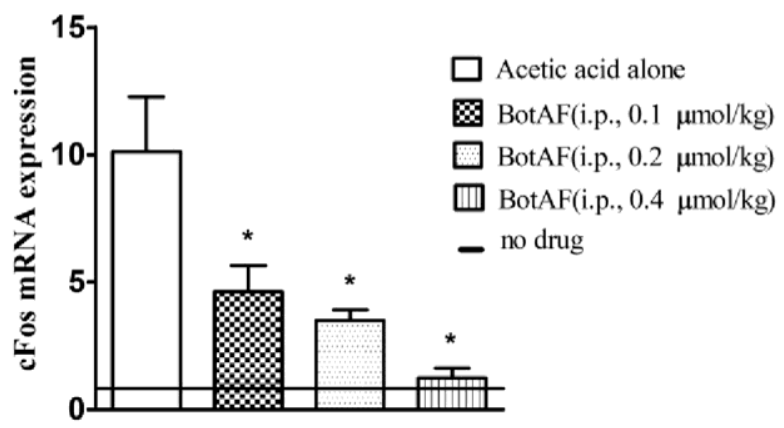

Treatment

B.1

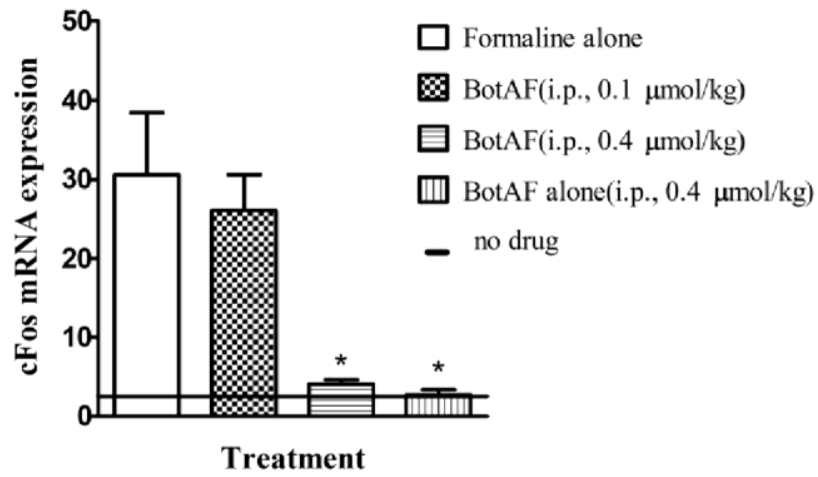

A.2

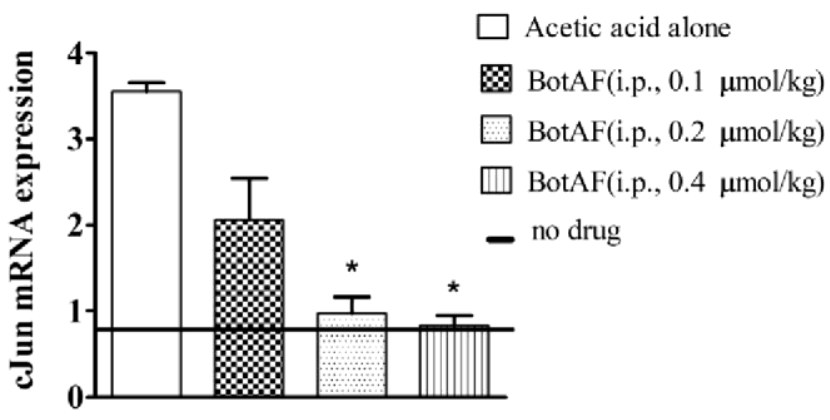

Treatment

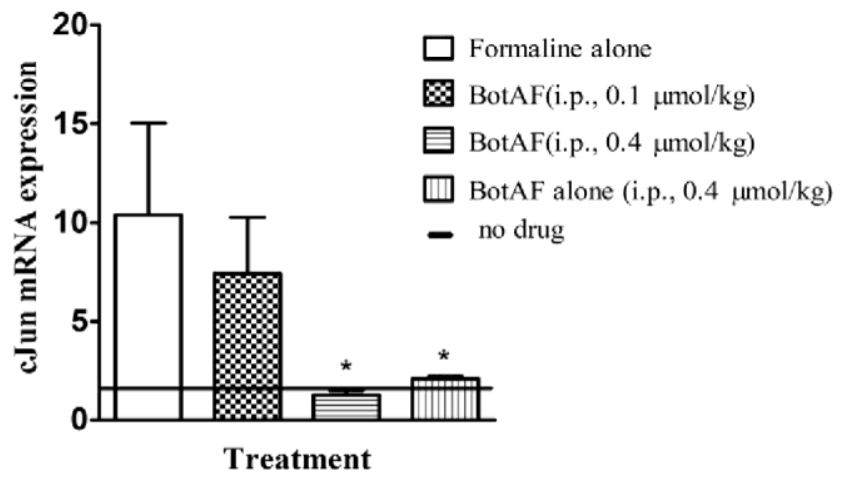

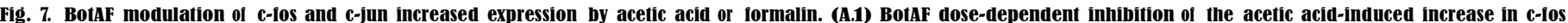

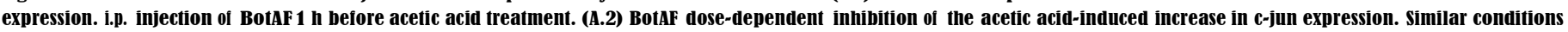

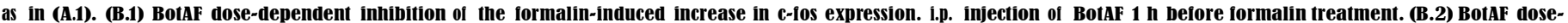

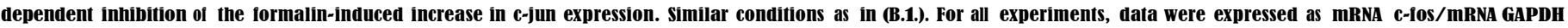
ratio or mRNA c-jun/mRNA GAPDH ratio.

evaluation of the extent of spinal mRNA inhibition levels of these oncogenes iurther confirms the analgesic potency of Botaf (previously demonstrated by in vivo ED50 values analysis) since it fully abolished their expression levels.

Finally, several experiments have been periormed to identify or rule out some specific molecular pharmacological targets of BotAF. We investigated some of the proposed targets of BotAF structural toxin analogues that were described to possess analgesic activity: beta like scorpion toxins and depressant insect toxins (Guan et al., 2001). Pharmacological targets of the b-like scorpion toxin family include TTX-resistant and TTX-sensitive voltage-gated $\mathrm{Na}^{\mathrm{b}}$ channels (Chen et al., 2006; Thu et al., 2009). Apart from Na ${ }^{\text {b }}$ channels, the group of Ji (Tan et al., 2001) showed that BmKAS1 inhibits $\left.I^{3} \mathbf{H}\right]-$ ryanodine binding on skeletal muscle by an indirect mechanism. Although $\mathrm{Na}^{\mathrm{b}}$ channels constitute more relevant pharmacological targets for analgesic activity than ryanodine receptors, it should be stressed out that b-like scorpion toxins have weak affinities for $\mathrm{Na}^{\mathbf{b}}$ channels. In fact, in brain synaptosomes, BotaF binds onto a weak affinity target, which is coherent with its lack of i.c.v. toxicity. We have also detected a limited activity of BotaF towards voltage-gated $\mathrm{Na}^{\mathrm{b}}$ channels of DRG neurons and no activity at all onto ryanodine receptors. A more profound block of TTX-resistant Na ${ }^{b}$ channels was found suggesting that among the various members of voltagegated $\mathrm{Na}^{\mathrm{b}}$ channels, BotaF may preferentially target TTX-resistant $\mathrm{Na}^{\mathrm{b}}$ channels, although these findings are not very conclusive for the moment. It should be noted that the DRG neuron TTX-R sodium channels has been demonstrated to be the most promising targets for analgesics (Silos-Santiago, 2008). Although our results are coherent with the effects of other members of the same family of toxins, the weak level of current inhibition and the relatively low affinity for this efiect questions whether voltage-gated $\mathrm{Na}^{\mathrm{b}}$ channels are the real pharmacological target whereby the potent analgesic efiect of Botaf occurs. While several studies implicate ryanodine receptors in nociception, BotaF obviously lacks the ability to bind to ryanodine receptors contrary to the homologous toxins, BmKAs and AS1. For the depressant insect toxins group, another mechanism of anti-nociception was proposed: release of endogenous opioids. This is also not the case of Botaf where naloxone fails to antagonize the analgesic activity of BotAF. Thus, we conclude that more detailed investigations are warranted to identify the real pharmacological targets of BotAF.

With regard to the analgesic efiects of BotAF, several features are worth discussion. First, BotaF is active on acute somatic pain (hotplate and tail-flick models), on inflammatory somatic pain (formalin test), and on inflammatory visceral pain (writhing test). This indicates that BotAF acts through molecular targets that play a key role in conduction and transmission of different nociceptive information rather than on pain transduction. Second, the mode of action of Botaf does not involve opioid receptors or an AINS-like mechanism, clearly distinguishing this molecule from these 
A.

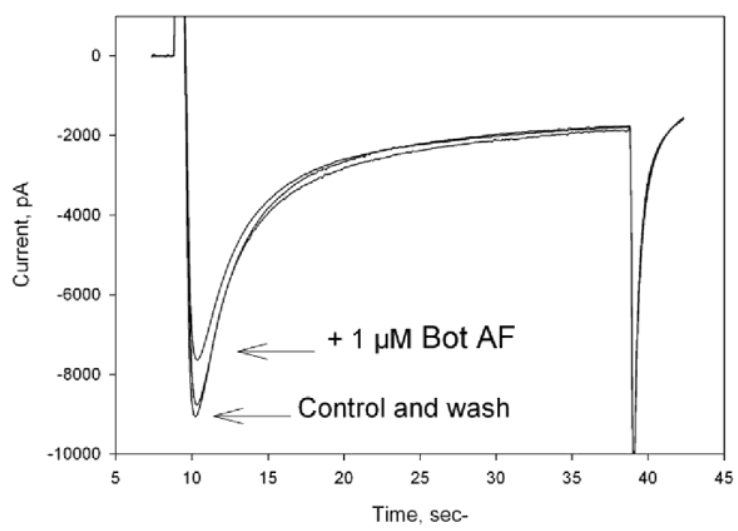

B.

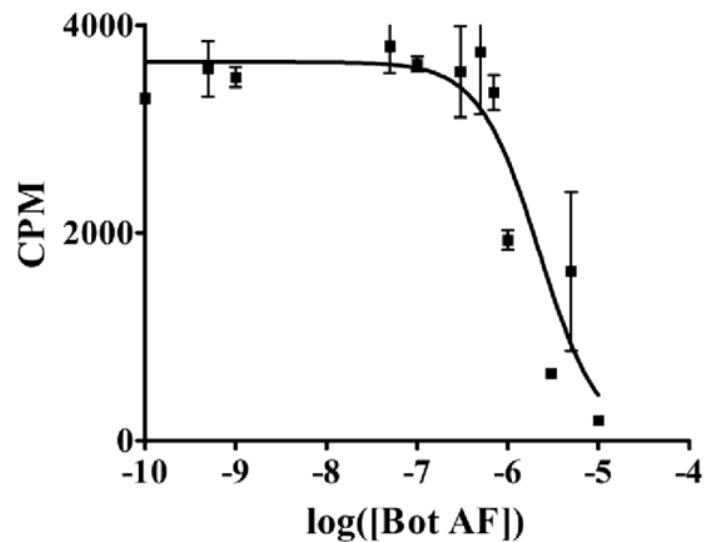

C.

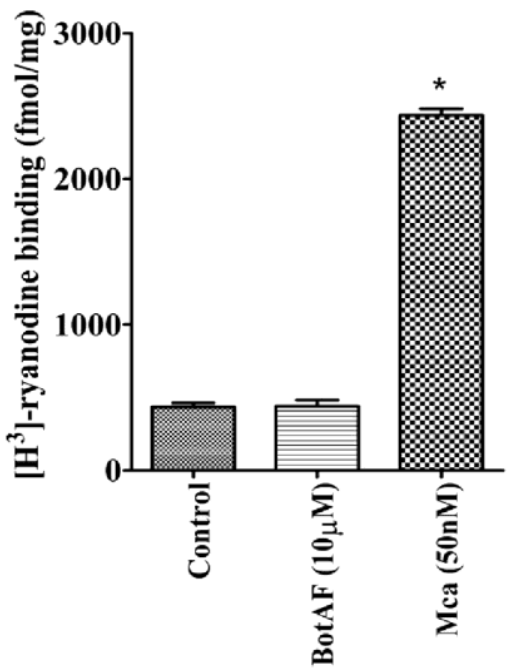

Treatment

Fig. 8. Pharmacology of Botaf. (A) Representative effect of $1 \mathrm{mM}$ BotaF on voltagegated Na ${ }^{b}$ currents from isolated DRG neurons and mean inhibition. (B) competitive displacement of $\mathrm{I}^{125} \mathrm{II}-\mathrm{BotaF}$ binding by unlabeled BotAF onto rat brain synaptosomes. (C) Effect of BotaF on [ $\left.{ }^{3} \mathrm{H}\right]$-ryanodine binding compared to the effect of maurocalcine.

common classes of analgesics. Third, the observation that BotaF prevents the increase in c-jun or c-ios under various pain stimuli is indicative that BotaF prevents the electrical activity of nociceptive neuronal aiferents. This observation is in line with the data
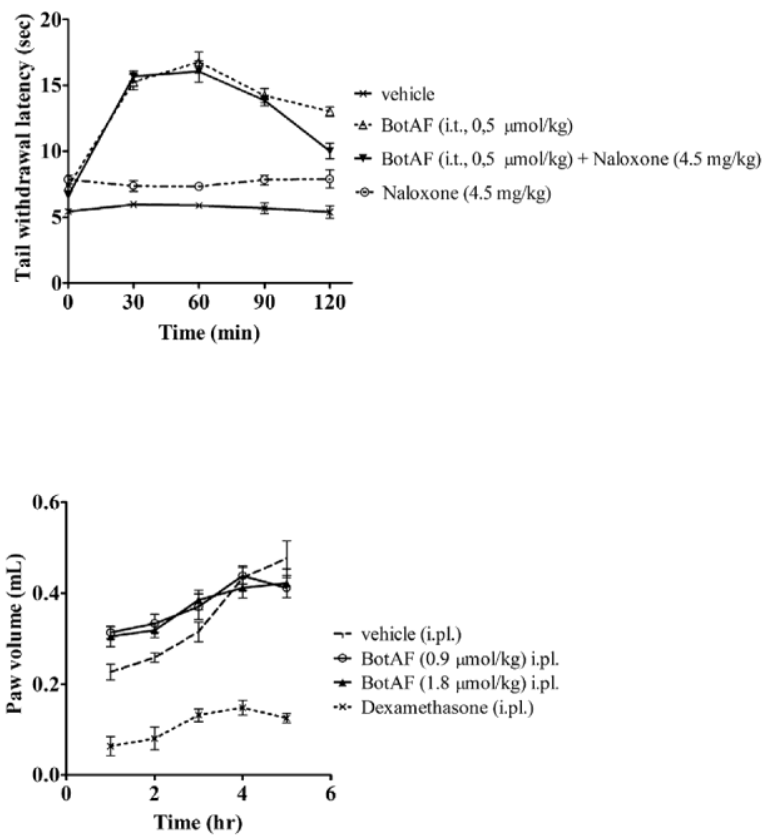

Fig. 9. BotaF does not act through an opioid receptor and has no anti-infiammatory activity. (A) Naloxone and naloxone methiodide modulation of BotaF antinociceptive effect in the tail-flick test. (B) BotaF effect on the Carrageenan-induced oedema in the rat.

suggesting that the anti-nociceptive effect of BotAF is not mediated by a supra-spinal efiect. In that respect, it is informative that the anti-nociceptive efficacy of BotaF is reliant on the mode of administration. The i.c.v. route was not efficient for analgesia in all pain models used in this study. Conversely, i.t. injection of BotaF was the most efficient mode of administration, at least in the pain model tested (tail-flick model). The intraplantar injection revealed itself also very efficient for the formalin test. Similarly, the i.p. injection was found more potent for the writhing test than the other modes of administration. These data indicate that the peripheral action of BotaF is best when locally administered at the same compartment than where the nociception generates. Altogether, these observations argue in favor of a peripheral and/or spinal mechanism for BotAF activity.

\section{Acknowledgments}

We thank Pr. Haiedh Mejdoub (Faculty Sciences of Siax) and Dr .Srairi-Abid ('LR11IPT08 Venins et biomolecules therapeutiques, Tunis,) for their contribution to the toxin sequencing and characterization.

\section{References}

Abbadie, C., Besson, J.M., 1994. Chronic treatments with aspirin or acetaminophen reduce both the development of polyarthritis and Fos-like immunoreactivity in rat lumbar spinal cord. Pain 57 (1), 45e54. Apr.

Abbadie, C., Honore, P., Fournie-Zaluski, M.C., Roques, B.P., Besson, J.M., 1994. Efiects of opioids and non-opioids on c-Fos-like immunoreactivity induced in rat lumbar spinal cord neurons by noxious heat stimulation. Eur. J. Pharmacol. 258 (3), 215e227. Jun 13.

Abbott, F.V., Franklin, K.B., Westbrook, R.F., 1995. The formalin test: scoring properties of the first and second phases of the pain response in rats. Pain 60 (1), $01 \mathrm{e102}$.

Attarde, S.S., Pandit, S.V., 2016. Scorpion venom as therapeutic agent-current perspective. Int. J. Curr. Pharm. Rev. Res. 7 (2), 59e72. Review Article.

Bai, Z.T., Liu, T., Pang, X.Y., Chai, L.F., Ji, Y.H., 2007. Suppressíon by intrathecal BmK 
IT2 on rat spontaneous pain behaviors and spinal c-Fos expression induced by formalín. Brain Res. Bull. 73 (4e6), 248e253, 2007 Jul 12.

Barr, G.A., Limon, E., Luthmann, R.A., Barr, G.A., Cheng, J., Wang, S., 2003. Analgesia induced by local plantar injections of opiates in the formalin test in infant rats. Dev. Psychobiol. 42 (2), 111 e122. Mar.

Bianchí, R., Rezzani, R., Borsani, E., Rodella, L., 2003. mGlu5 receptor antagonist decreases fos expression in spinal neurons after noxious visceral stimulation. Brain Res. 960 (1e2), 263e266. Jan 17.

Chen, B., Ji, Y., 2002. Antíhyperalgesia efiect of BmK AS, a scorpion toxin, in rat by intraplantar injection. Brain Res. 952 (2), 322e326.

Chen, J., Feng, X.H., Shi, J., Tan, Z.Y., Bai, Z.T., Liu, T., Ji, Y.H., 2006. The antínociceptive effect of $\mathrm{BmK} \mathrm{AS}$, a scorpion active polypeptide, and the possible mechanism on specifically modulating voltage-gated Nap currents in primary afferent neurons. Peptides 27 (9), $2182 \mathrm{e} 2192$.

Collier, H.0.J., Dimneen, L.C., Johnson, C.A., Schneider, C., 1968. The abdominal constriction response and its suppression by analgesic drugs in the mouse. Br. J. Pharmacol. Chemother. 32, 295 e310.

Clot-Faybesse, 0., Devaux, C., Rochat, H., Guieu, R., 2001. In vivo neurotoxicity of Androctonus australis hector scorpion venom: evidence that the supra-thoracic nervous system is not implicated in the clinical manifestations. Toxicon 39 (7), 1003 e1007.

Cuil, Y., Song, Y.B., Ma, L., Liu, Y.F., Li, G.D., 2010. Site-directed mutagenesis of the toxin from the Chinese scorpion Buthus martensii Karsch (BmKAS): insight into sites related to analgesic activity. Arch. Pharm. Res. 33, 1633 e1639.

D'Amour, F.E., Smith, D.L., May 1941. A method for determining loss of pain sensation. J. Pharmacol. Exp. Therapeut. 72 (1), 74 e79.

Di ROSA, M., Sorrentino, L., 1968. The mechanism the inflammatory effect of carrageenin. Eur. J. Pharmacol. 4, 340e342.

Eckerskorn, c., Lottspeich, F., 1990. Combination of two-dimensional gel electrophoresis with microsequencing and amino acid composition analysis: improvement of speed and sensitivity in protein characterization. Electrophoresis 11 (7), $554 \mathrm{e} 561$.

Edman, P., Begg, G., 1967. A protein sequenator. Eur. J. Bíochem. 1, 80e91.

Escoubas, P., Bernard, C., Lambeau, G., Lazdunski, M., Darbon, H., 2003. Recombinant production and solution structure of PcTx1, the specific peptide inhibitor of ASIC1a proton-gated cation channels. Protein Sci. 12 (7), 1332 e1343.

Essack, M., Bajic, V.B., Archer, J.A., 2012. Conotoxins that confer therapeutic possibílities. Mar. Drugs 10 (6), 1244e1265. https://doi.org/10.3390/md10061244. Jun Epub 2012 Jun 4. Review.

Galeotti, N., Bartolini, A., Ghelardini, C., 2003. Diphenhydramine-induced amnesia is mediated by Gi-protein activation. Neuroscience $122,471 \mathrm{e} 478$.

Gordon, D., Ilan, N., Zillberberg, N., Gilles, N., Urbach, D., Cohen, L., Karbat, I., Froy, 0. Gaathon, A., Kallen, R.G., Benveniste, M., Gurevitz, M., 2003. An 'old World scorpion beta-toxin that recognizes both insect and mammalian sodium channels. Eur. J. Biochem. 270 (12), 2663 e2670.

Gray, E.G., Whittaker, V.P., 1962. The isolation of nerve endings from brain: an electron-microscopic study of cell fragments derived by homogenization and centrifugation. J. Anat. 96, $79 \mathrm{e} 88$.

Guan, R., Wang, C.G., Wang, M., Wang, D.C., 2001. A depressant insect toxin with a novel analgesic efiect firom scorpion Buthus martensii Karsch. Bíochìm. Bĩophys. Acta 1549, 9e18.

Hall, T.A., 1999. BioEdit: a user-friendly biological sequence alignment edítor and analysis program for Windows 95/98/NT. Nucleic Acids Symp. Ser. 41, 95e98.

Herzog, R.I., Cummins, T.R., Ghassemi, F., Dib-Hajj, S.D., Waxman, S.G., 2003. Distinct repriming and close-state inactivation kinetics of Nav1.6 and Nav1.7 sodium channels in mouse spinal sensory neurons. J. Physiol. 551.

Jen6, P., Miní, T., Moes, S., Hintermann, E., Horst, M., 1995. Internal sequences from proteins digested in polyacrylamide gels. Anal. Biochem. 224 (1), $75 e 82$.

Jensen, J.E., Cristofori-Armstrong, B., Anangi, R., Rosengren, K.J., Lau, C.H., Mobli, M., Brust, A., Alewood, P.F., King, G.F., Rash, L.D., 2014. Understanding the molecular basis of toxin promiscuity: the analgesic sea anemone peptide APETx2 interacts with acid-sensing ion channel 3 and hERG channels via overlapping pharmacophores. J. Med. Chem. 57 (21), 9195 e9203.

Jones, B.J., Roberts, D.J., 1968. The quantitative measurement of motor incoordination in naive mice using an accelerating rotarod. J. Pharm. Pharmacol. 20, 302 e304.

Lan, Z.D., Daí, L., Zhuo, X.L., Feng, J.C., Xu, K., Chi, C.W., 1999. Gene cloning and sequencing of BmK AS and BMK AS-1, two novel neurotoxins from the scorpion Buthus martensi Karsch. Toxicon 37 (5), 815e823.
Liu, T., Pang, X.Y., Jiang, F., Bai, Z.T., Ji, Y.H., 2008. Anti-nociceptive effects induced by intrathecal injection of $\mathrm{BmK} \mathrm{AS}$, a polypeptide from the venom of chinesescorpion Buthus martensi Karsch, in rat formalin test. J. Ethnopharmacol. 117 (2), 332e338. May 8.

Martin-Eauclaire, M.F., Abbas, N., Sauze, N., Mercier, L., Berge-Leiranc, J.L., Condo, J., Bougis, P.E., Guieu, R., Sep 20 2010. Involvement of endogenous opioid system in scorpion toxin-induced antinociception in mice. Neuroscí. Lett. 482 (1), 45 e50.

Loret, E.P., Martin-Eauclaire, M.F., Mansuelle, P., Sampierī, F., Granier, C., Rochat, H., Jan 22 1991. An anti-insect toxin purified from the scorpion Androctonus australis Hector also acts on the alpha- and beta-sites of the mammalian sodium channel: sequence and circular dichroism study. Biochemistry 30 (3), 633e640.

Marty, D., Thevenon, $\mathbf{C}$, Scotto, $\mathbf{S}$, Groh, $\mathbf{S}$, Sainnier, M., Robert, D., Grunwald, $\mathbf{M}$. 2000. Cloning and characterization of a new isoform of skeletal muscle triadin. J. Bíol. Chem. 275, 8206e8212.

Mestre, C., Pelissier, T., Fialip, J., Wilcox, G., Eschalier, A.A., 1994. Method to periorm direct transcutaneous intrathecal injection in rats. J. Pharmacol. Toxicol. Meth. 32 (4), $197 \mathrm{e} 200$.

Miranda, F., Kupeyan, C., Rochat, H., Rochat, C., Lissítzky, S., 1970. Purification of animal neurotoxins. Isolation and characterization of four neurotoxins from two different sources of Naja haje venom. Eur. J. Biochem. 17 (3), 477 e484.

Morgan, J.I., Curran, T., 1991. Stimulus-transcription coupling in the nervous system: involvement of the inducible proto-oncogenes fos and jun. Annu. Rev. Neurosci. 14, 421 e451. Review.

ozkan, 0., Ciiftcí, G., Pekmezcí, G.L., Kar, S., Uysal, H., Karaer, K.L., 2007. Proteíns, lethality and in vivo effects of Iurus dufoureius asiaticus scorpion venom. Toxicon 50 (3), 394e399. Sep 1.

Pipelzadeh, M.H., Jalalí, A., Deziulian, A.R., Khorasganí, Z.N., Sarvestaní, S., Ghalambor, A.H., Azarpanah, A., 2015. A forward to optimization of antivenom therapy: an in vivo study upon the effectiveness of the antivenom against early and delayed nephrotoxicity induced by the venom of the Iranian scorpion Hemiscorpius lepturus in rat. Toxicon 100, 13 e19 jun15.

Plummer, John L., Cmielewski, Patricia L., Gourlay, Geofirey K., owen, Harry, Cousins, Michael J., 1991. Assessment of antinociceptive drug effects in the presence of impaired motor performance. J. Pharmacol. Meth. 26 (1), $79 e 87$.

Pu, X.C., Wong, P.T., Gopalakrishnakone, P., 1995. A novel analgesíc toxîn (hannalgesin) from the venom of king cobra (ophiophagus hannah). Toxicon 33 (11), 1425e1431. Nov.

Rauck, R.L., Wallace, M.S., Burton, A.W., Kapural, L., North, J.M., 2009. Intrathecal ziconotide for neuropathic pain: a review. Pain Pract. 9 (5), 327e337. Sep-0ct.

Rochat, H., Rochat, C., Miranda, F., Lissitzky, S., Edman, P., 1970. The amino acid sequence of neurotoxin I of Androctonus australis hector. Eur. J. Biochem. 17 (2), $262 \mathrm{e} 266$.

Shao, J.H., Wang, Y.Q., Wu, X.Y., Jiang, R., Thang, R., Wu, C.F., Zhang, J.H., 2008. Cloning, expression and pharmacological activity of BmK AS, an active peptide from scorpion Buthus martensii Karsch. Biotechnol. Lett. 30 (1), 23 e29.

Silos-Santiago, I., Jan 2008. The role of tetrodotoxin-resistant sodium channels in pain states: are they the next target for analgesic drugs? Curr. opin. Investig. Drugs 9 (1), 83e89. Review.

Tan, Z.Y., Mao, X., Xiao, H., Zhao, Z.Q., Ji, Y.H., 2001. Buthus martensí Karsch agonist of skeletal-muscle RyR-1, a scorpion active polypeptide: antinociceptive efiect on rat peripheral nervous system and spinal cord,and inhibition of voltagegated Na(b) currents in dorsal root ganglion neurons. Neurosci. Lett. 297 (2), 65 e68.

Thompson, J.D., Higgins, D.G., Gibson, T.J., 1994. CLUSTAL W: improving the sensitivity of progressive multiple sequence alignment through sequence weighting, position-specífic gap penalties and weỉght matrix choice. Nucleic Acíds Res. 22 (22), $4673 \mathrm{e} 4680$.

Wang, Y., Wang, L., Cuí, Y., Song, Y.B., Liu, Y.F., Zhang, R., Wu, C.F., Zhang, J.H., 2011. Purícation, characterization and functional expression of a new peptide with an analgesic effect from Chinese scorpion Buthus martensii Karsch (BmK AGPSYPU1). Biomed. Chromatogr. 25 (7), 801 e807.

Warren, P.H., Ison, J.R., 1982. Selective action of morphine to nociceptive stimulation in the rat: a contribution to the assessment of analgesia. Pharmacol. Biochem. Behav. 16, 869 e874.

Zhu, M.M., Tao, J., Tan, M., Yang, H.T., Ji, Y.H., 2009. U-shaped dose-dependent effects of BMKAS, a unique scorpion polypeptide toxin, on voltage-gated sodium channels. Br. J. Pharmacol. 158 (8), 1895 e1903. 